\title{
Weakly nonlinear nonaxisymmetric oscillations of capillary bridges at small viscosity
}

\author{
María Higuera, José A. Nicolás, and José M. Vega \\ E.T.S.I. Aeronauticos, Universidad Politécnica de Madrid, Plaza Cardenal Cisneros. \\ 3. 28040 Madrid, Spain
}

\begin{abstract}
Weakly nonlinear nonaxisymmetric oscillations of a capillary bridge are considered in the limit of small viscosity. The supporting disks of the liquid bridge are subjected to small amplitude mechanical vibrations with a frequency that is close to a natural frequency. A set of equations is derived for accounting the slow dynamics of the capillary bridge. These equations describe the coupled cvolution of two counter-rolating capillary waves and an associated streaming flow. Our derivation shows that the effect of the streaming flow on the capillary waves cannot be a priori ignored because it arises at the same order as the leading (cubic) nonlinearity. The system obtained is simplified, then analyzed both analytically and numerically to provide qualitative predictions of both the relevant large time dynamics and the role of the streaming flow. The case of parametric forcing at a frequency near twice a natural frequency is also considered.
\end{abstract}

\section{INTRODUCTION AND FORMULATION}

Capillary jets and bridges are basic configurations to analyze capillary effects and were first considered by Plateau ${ }^{1,2}$ and Rayleigh, ${ }^{3,4}$ using the previous basic works on capillary interfaces by Young ${ }^{5}$ and Laplace. ${ }^{6}$ Capillary bridges are of interest in materials processing ${ }^{7}$ and in the experimental measurement of viscosity and surface tension, ${ }^{8}$ and have becn observed in various plysical processes of industrial interest such as flow through porous media and particulates agglomeration. ${ }^{10}$ In particular, mechanical vibrations of capillary bridges are of interest in material processing in microgravity because the float zone modeled by the capillary bridge is subject to various oscillatory excitations resulting from on board machinery and crew maneuvering, and have been the object of numerous works in the last thirty years. But unfortunately most of these works dealt with either the linear approximation (see Ref. 11 for references) and/or one-dimensional approximations, ${ }^{12}$ whose validity is restricted to slender bridges and cannot be used in the nonaxisymmetric case. Nonlinear effects have been only considered in the strictly axisymmetric case in a few works, most of them based on direct numerical simulations, which become quite expensive as viscous effects decrease. ${ }^{1.3}$

A weakly nonlinear analysis of resonant, axisymmetric oscillations of capillary bridges at small viscosity and small driving amplitude was performed by two of us. ${ }^{14,15}$ The outcome in Ref. 14 was an amplitude equation for the complex amplitude of the excited capillary wave, which is standing. In addition, the mean flow produced by the wave was calculated in order to elucidate its ability to counterbalance the undesirable thermocapillary flow, ${ }^{16}$ which is always present in nonisothermal conditions; this counterbalancing effect has been analyzed both experimentally ${ }^{17}$ and theoretically. ${ }^{18.19}$ The mean flow produced by the capillary waves was the well known streaming flow ${ }^{20}$ (also called stcady streaming, or acoustic streaming ${ }^{21}$ ), driven by nonzero time averaged Reynolds stresses in the Stokes boundary layer attached to the disks supporting the bridge.

The present paper could be considered as the extension of Ref. 14 to the nonaxisymmetric case, but this extension is far from straightforward due to the role of the streaming flow, which does not affect the capillary wave dynamics (at least at leading order) in the axisymmetric casc. ${ }^{14}$ In the nonaxisymmetric case instead the capillary wave dynamics are coupled to the streaming flow evolution, which makes the weakly nonlinear dynamics both more involved and subtle. The same coupling effect of the mean flow has been recently demonstrated in related problems. ${ }^{22} \mathrm{~A}$ second novel property of the mean flow is that it is also driven by the oscillatory boundary layer attached to the free surface provided that the capillary wave is not of the standing type, see below.

We consider (see Fig. 1) a capillary bridge held by surface tension between two parallel, circular, coaxial disks of equal radii $R^{*}$, at a distance $L^{*}$ apart from each other, which exhibits a rigid-solid, harmonic oscillation with an amplitude $a^{*} \ll R^{*}$ and a frequency $\omega^{*}$, assumed to be close to a natural frequency of the bridge. The volume of the liquid equals that of the space in the cylinder bounded by the unperturbed disks, and the free surface is assumed to be anchored at the edges of the disks. The density $\rho$ and the kinematic viscosity $\nu$ of the liquid, and the surface Iension coefficient $\sigma$ are constant, and such that the capillary number (also called Ohnesorge number)

$$
C=\nu p^{1 / 2} /\left(\sigma R^{*}\right)^{1 / 2} \ll 1
$$

In addition we neglect both gravity and the surrounding air, and use $R^{*}$ and the capillary time $\left(\rho R^{* 3} / \sigma\right)^{1 / 2}$ as characteristic length and time for nondimensionalization. The continuity and momentum conservation equations in an oscillating 


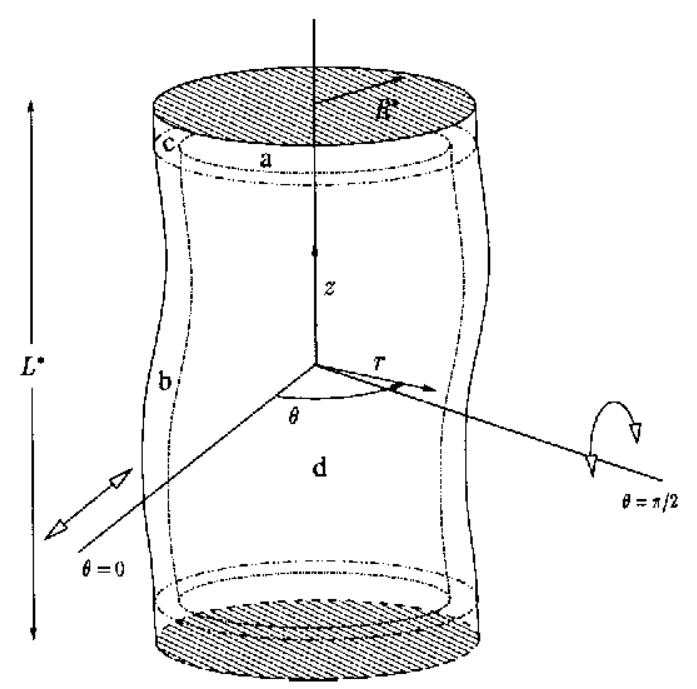

FIG. 1. Sketeh of the eapillary liquid bridge, which is subject to monochromatic, solid rigid oscillation consisting of a beating around a horizontal axis and a lateral oscillation, with nondimensional frequency os and amplitudes $\alpha \mu$ and $\alpha(1-\mu)$, respectively. The additional nondimensional parameters are the Ohnesorge number $C=\nu \rho^{1 / 2} /\left(\sigma R^{*}\right)^{1 / 2}$ and the slendemess $A$ $L^{*} /\left(2 R^{*}\right)$, where $\nu$ is the kinematic viscosity, $\rho$ is the density, and $\sigma$ is the surface tension. The evolution equations below for the streaming flow apply in the bulk (d), outside the oscillatory boundary layers (a) and (b) and the corner tori $(c)$, whose nondimensional size is $O\left(c^{1 / 2}\right)$.

cylindrical coordinate system attached to the disks, $(r, \theta, z)$ (with associated unil vectors $e_{r}, e_{\ell}$, and $e_{z}$ ) are

$\nabla \cdot \boldsymbol{v}-0$.

$\partial \boldsymbol{v} / \partial t-\boldsymbol{v} \times(\nabla \times \boldsymbol{v})-\nabla q+C \Delta \boldsymbol{v}+a \boldsymbol{h}+O\left(a|\boldsymbol{v}|+a^{2}\right)$,

in $-\Lambda<z<\Lambda, 0<r<f(\theta, z, l)$, where $v$ is the velocity, $q$ is the stagnation pressure ( - pressure $+|\boldsymbol{v}|^{2} / 2$ ), $\Lambda$ $=L^{*} /\left(2 R^{*}\right)$ is the slendemess and $f$ is the radial deflection of the free surface. The nondimensional forcing amplitude is

$$
a \equiv a^{*} / R^{*} \ll 1
$$

and the vector $h$ is given by

$$
\begin{aligned}
& \boldsymbol{h}-\left[\mu\left(z \cos \theta \boldsymbol{e}_{r}-z \sin \theta \boldsymbol{e}_{\theta}-r \cos \theta \boldsymbol{e}_{z}\right)\right. \\
& \left.\mid\left(\begin{array}{lll}
1 & \mu
\end{array}\right)\left(\cos \theta \boldsymbol{e}_{r} \quad \sin \theta \boldsymbol{e}_{\theta}\right)\right] \omega^{2} \cos \omega t,
\end{aligned}
$$

where $\omega-\omega^{*}\left(\rho R^{* 3} / \sigma\right)^{1 / 2}$ is the nondimensional forcing frequency, and the real coefficient $\mu$ is such that $0<\mu<1$. The body force $a \boldsymbol{h}+O\left(a|\boldsymbol{v}|+a^{2}\right)$ results from (i) a beating around the $\theta=\pi / 2, z=0$ axis (if $\mu=1$ ), and (ii) a lateral oscillation along the $\theta-0, z-0$ axis (if $\mu-0)$; for simplicity both motions are assumed to be in phase. These are simple devices to get a direct forcing that preserves the reflection symmetry on the plane $\theta-0$ but breaks invariance under rotation: the beating also breaks the reflection symmetry on the $z=0$ plane, which is convenient to force those eigenmodes that are odd in the vertical coordinate (see Fig. 1). The boundary conditions resulting from nonslipping at the disks, kincmatic compatibility and tangential and normal stress balances at the free surface are

$$
\begin{aligned}
& \boldsymbol{v}=\boldsymbol{0}, \quad f=1 \quad \text { at } z= \pm \Lambda, \\
& \boldsymbol{v} \cdot \boldsymbol{n}-(\partial f / \partial t)\left(\boldsymbol{c}_{r} \cdot \boldsymbol{n}\right), \quad \text { at } r-f,
\end{aligned}
$$

$$
\begin{aligned}
& (\mathcal{T} \cdot \boldsymbol{n}) \times \boldsymbol{n}-\boldsymbol{0} \\
& q|\boldsymbol{v}|^{2} / 2 \mid 2 M=2 C(\mathcal{T} \cdot \boldsymbol{n}) \cdot \boldsymbol{n} \quad \text { at } r=f .
\end{aligned}
$$

Here $\mathcal{T}-\left[\nabla \boldsymbol{v}+(\nabla \boldsymbol{v})^{\top}\right] / 2$ is the (rate of) strain tensor (i.e., the symmetric part of $\nabla \boldsymbol{v}$ ) and the /aplacian of $\boldsymbol{v}$ is defined as usually (recall that the flow is incompressible), $\Delta v-2 \nabla$ $\mathcal{T} \equiv \nabla \times(\nabla \times \boldsymbol{v}) ; \boldsymbol{n}$ and $M$ are the outward unit normal and the mean curvature of the free surface, respectively. For convenience we recall that the volume is conserved, namely

$$
\int_{-\Lambda}^{\Lambda} \int_{0}^{2 \pi} f^{2} d \theta d z-4 \pi \Lambda
$$

In Sec. II we shall perform a weakly nonlinear, multiscale analysis in the limit (1), (4). The velocity and the stagnation pressure in the bulk (outside the boundary layers) and the free surface deflection will be given by [cf. (22) and (23) below]

$$
\begin{gathered}
\boldsymbol{v}=\boldsymbol{\varepsilon} \mathrm{e}^{\mathrm{i} \omega t}\left[A(\tau) \boldsymbol{V}_{i,}^{-}(r, z) \mathrm{e}^{\mathrm{i} \theta} \mid B(\tau) \boldsymbol{V}_{0}^{-}(r, z) \mathrm{e}^{\mathrm{i} \theta}\right] \\
+ \text { c.c. }+\varepsilon^{2} \boldsymbol{v}_{2}^{s}(r, \theta, z, \tau)+\cdots, \\
\left(\begin{array}{rl}
q \quad 1, f \quad 1)= & \varepsilon\left(Q_{0,}, F_{0}\right) \mathrm{e}^{\mathrm{i} \omega t}\left[A(\tau) \mathrm{e}^{\mathrm{i} \theta} \mid B(\tau) \mathrm{e}^{-\mathrm{i} \theta}\right] \\
& + \text { c.c. }+\cdots,
\end{array}\right.
\end{gathered}
$$

where

$$
0<\varepsilon<1
$$

is the capillary wave steepness,

$$
\tau=\varepsilon^{2} t
$$

is a slow time variable, and c.c. stands for complex conjugate. The $O(\varepsilon)$ terms account for a pair of counter-rotating capillary waves with complex amplitudes $A$ and $B$, and $\varepsilon^{2} v_{2}^{s}$ is the streaming flow velocity. The capillary waves and the streaming flow are both nearly marginal, namely they cxhibit a small damping rate and thus none of them can be ignored $a$ priori. The capillary waves are associated with the capillary (or imviscid) eigenmodes, $\left(V_{0}^{\dot{*}}, Q_{0}, F_{0}\right) \mathrm{e}^{ \pm \mathrm{i} \theta}$, which exhibit an eigenfrequency $\Omega \neq 0$, assumed to be simple and close to the forcing frequency. The remaining unforced capillary modes decay by viscous dissipation and can be ignored. We are implicitly excluding second and third order internal resonances, which would appear if either $\Omega=\Omega_{1} \pm \Omega_{2}$ and 1 $-m_{1} \pm m_{2}$, or $\Omega-\Omega_{1} \pm \Omega_{2}+\Omega_{3}$ and $1-m_{1} \pm m_{2}+m_{3}$, where $\Omega_{1}, \Omega_{2}$, and $\Omega_{3}$ are natural frequencies, not necessarily different from each other and from $\Omega$, and $m_{1}, m_{2}$, and $m_{3}$ are the associated wave numbers. The streaming flow is associated with the hydrodynamic (or viscous) eigenmodes, which cxhibit a $O(C)$ damping rate; ${ }^{11}$ after rescaling, these eigenmodes are the solutions of Eqs. (C2)-(C4) in Appendix $\mathrm{C}$. Both the complex amplitudes and the streaming flow velocity depend weakly on time. We anticipate that the amplitude equations are 


$$
\begin{aligned}
\frac{d A}{d \tau}= & \left(d / \varepsilon^{2} \mid \mathrm{i} \delta / \varepsilon^{2}\right) A \mid \mathrm{i}\left(\left.\alpha_{3}|A|^{2}\left|\alpha_{4}\right| B\right|^{2}\right) A \\
& \mathrm{i} \alpha_{5} \int_{\Sigma_{0}} \boldsymbol{g} \cdot \boldsymbol{v}_{2}^{s} d \boldsymbol{x} A \mid \mathrm{i} \alpha_{6} a / \varepsilon^{3}, \\
\frac{d B}{d \tau}- & -\left(d / \varepsilon^{2}+\mathrm{i} \delta / \varepsilon^{2}\right) B+\mathrm{i}\left(\alpha_{3}|B|^{2}+\alpha_{4}|A|^{2}\right) B \\
+ & \mathrm{i} \alpha_{5} \int_{\Sigma_{0}} \boldsymbol{g} \cdot \boldsymbol{v}_{2}^{s} d \boldsymbol{x} B+\mathrm{i} \alpha_{6} a / \varepsilon^{3},
\end{aligned}
$$

where $\Sigma_{(0)}:-\Lambda<z<\Lambda, 0<r<1$ is the unperturbed capillary bridge, and the streaming flow is given by

$$
\begin{aligned}
& \nabla \cdot \boldsymbol{v}_{2}^{s}=0 \text { in } \Sigma_{0}, \\
& \partial \boldsymbol{v}_{2}^{s} / \partial \tau-\left[\boldsymbol{v}_{2}^{s}+\left(|B|^{2}-|A|^{2}\right) \boldsymbol{g}\right] \times\left(\nabla \times \boldsymbol{v}_{2}^{s}\right) \\
& --\nabla \widetilde{q}^{s}+\left(C / \varepsilon^{2}\right) \Delta \boldsymbol{v}_{2}^{s} \text { in } \Sigma_{0} \text {, }
\end{aligned}
$$

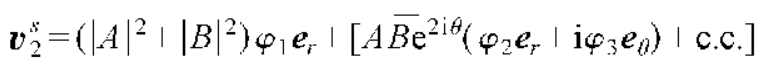

$$
\begin{aligned}
& \text { । }\left(|B|^{2} \quad|A|^{2}\right) \varphi_{4} e_{\theta} \quad \text { at } z= \pm \Lambda \text {, } \\
& \boldsymbol{v}_{2}^{s} \cdot \boldsymbol{e}_{r}-0,\left(\partial \boldsymbol{v}_{2}^{s} / \partial r-\boldsymbol{v}_{2}^{s}\right) \cdot \boldsymbol{e}_{\emptyset}-\left(|\beta|^{2}-|A|^{2}\right) \varphi_{5}, \\
& \left(\partial \boldsymbol{v}_{2}^{*} / \partial r\right) \cdot \boldsymbol{e}_{z}=0 \text { at } r=1 \text {. }
\end{aligned}
$$

Here the coefficients $\alpha_{3}, \ldots, \alpha_{6}$ are real as are the functions $g, \varphi_{j}=\varphi_{j}(r)$ for $j=1, \ldots, 4$ and $\varphi_{s}=\varphi_{s}(z)$. $\widetilde{q}^{s}$ is an appropriatc short-lime-averaged modified pressurc, and

$$
d=\alpha_{1} C^{1 / 2} \mid \alpha_{2} C \text { and } \delta=\alpha_{1} C^{1 / 2} \mid \omega \quad \Omega
$$

are the damping rate and detuning (with respect to the natural frequency $\Omega$ ) of the capillary waves and satisfy

$$
0<d \ll 1 \text { and }|\delta| \ll 1 \text {. }
$$

Equations (12)-(17) are the coupled amplitudestreaming flow (CASF) equations, which give the weakly nonlincar cvolution of two forced counter-rotating waves and the associated streaming flow. Some remarks are in order:

(a) The CASF equations are invariant under the symmetry

$$
A \leftrightarrow B, \quad \theta \longrightarrow-\theta,
$$

$\boldsymbol{v}-u e_{r}+v e_{\theta}+w e_{z} \rightarrow u e_{r}-v e_{\theta}+w e_{z}$,

which results from invariance of the original equations under reflection on the plane $\theta-0$. Thus, only one of the amplitude equations needs to be derived.

(b) In the amplitude equations (12) and (13) we are considering only the leading-order effects of inertia, nonlinearity, coupling with the streaming flow and forcing. but we are taking a sccond-order approximation of the damping rate and detuning [see Eq. (18)]. In so doing. we are taking into account that $\alpha_{2} / \alpha_{1}$ is typically fairly large. " Thus, if the $O(C)$-term were neglected. the resulting approximation of the damping rate would be good only for extremely small values of $C$ (say, $C$ $\leqslant 10^{5}$ ); the second-order approximation, instead. gives good results for $C \leqslant 10^{-2}$, in capillary bridges ${ }^{11}$ and also in related problems associated with gravitycapillary waves in finite containers. ${ }^{23}$ (c) In order to avoid unnecessary restrictions, the small parameters $C, \delta, \varepsilon$, and $a$ will be treated as independent parameters when deriving the CASF equations. After completing the derivation these parameters will be related to each other in Sec. III in such a way that as many terms as possible are comparable in the equations; this will give a distinguished limit. ${ }^{24}$

(d) Since both $\alpha_{5}$ and $\boldsymbol{g}$ are real, that term accounting for coupling to the streaming flow is conservative in (12) and (13), namely it does not affect the evolution of the rc-scaled time-averaged energy (at leading order), $|A|^{2}+|B|^{2}$. This is consistent with the fact that the contribution of the streaming flow to the time-averaged (kinelic) energy is higher order, $O\left(\varepsilon^{4}\right)$, compared to that associated with the capillary waves, which is $O\left(\varepsilon^{2}\right)$.

(e) The applied forcing is reflection-symmetric in the azimuthal coordinate, which prohibits a preference for one counter-rolating wave over the other. Thus the coeflicients of the direct forcing term are the same in Eqs. (12) and (13). Different forcing coefficients result from applying non rellection-symmetric forcing. Also, bccause of the nature of the forcing device [see Eq. (5)]. only those modes with azimuthal wave number $m=1$ can be effectively excited. The remaining modes can be excited by parametric forcing, which is also considcred.

With these ideas in mind, this paper is organized as follows. The CASF equations are derived in Sec. II for the simplest case considered above in which the capillary waves are directly excited by external vibrations at a frequency close to a natural frequency. Because of the threedimensional (3D) Navier-Stokes-type equation (15), the numerical treatment of the CASF equations is quite expensive and outside the scope of this paper. Thus in Sec. III we present a hierarchy of simplified equations, which are analyzed to obtain several predictions on the relevant dynamics, with special emplasis on the role of the streaming flow to either enhance or prevent complexity, beyond the simplest. reflection-symmetric standing waves. The case of parametric forcing is considered in Sec. IV and the conclusions are drawn in Sec. V.

\section{DERIVATION OF THE CASF EQUATIONS}

We consider Eqs. (2), (3), (6)-(8) in the limit

$$
C \ll 1, \quad|\omega-\Omega| \ll 1, \quad \varepsilon \ll 1 .
$$

Since $C$ is small (viscous effects are weak and) the capillary bridge exhibits four distinct regions (see Fig. 1): (a) Two Stokes boundary loyers attached to the disks; (b) an interface boundary layer attached to the free surface; (c) two small comer tori near the rim of the disks, whose analysis is not needed below; and (d) the bulk, which is the remaining part of the capillary bridge. The size of regions (a), (b) and (c) (where inertia and viscous terms are comparable in momentum equation) is $O\left(C^{1 / 2}\right)$. 
We seek the following expansions for the velocity and the stagnation pressure in the bulk, and the free surface deflection

$$
\begin{gathered}
\boldsymbol{v}-\boldsymbol{\varepsilon} \mathrm{e}^{\mathrm{i} \omega t}\left[A(\tau) \boldsymbol{v}_{0}^{+}(r, \theta, z)+B(\tau) \boldsymbol{v}_{0}^{-}(r, \theta, z)\right] \\
+ \text { c.c. }+\varepsilon C^{1 / 2} \boldsymbol{v}_{1}+\varepsilon^{2} \boldsymbol{v}_{2}+\varepsilon C \boldsymbol{v}_{3}+\varepsilon^{2} C^{1 / 2} \boldsymbol{v}_{4} \\
\left|\boldsymbol{\varepsilon}^{3} \boldsymbol{v}_{5}\right| a \boldsymbol{v}_{6} \mid \cdots, \\
(q-1, f-1)-\varepsilon \mathrm{c}^{\mathrm{i} \omega t}\left[A(\tau)\left(q_{0}^{\prime}, f_{0}^{\prime}\right)+B(\tau)\left(q_{0}, f_{0}\right)\right] \\
+ \text { c.c. }+\cdots,
\end{gathered}
$$

where the expansions for $q-1$ and $f-1$ are similar to that for $\boldsymbol{v}$. The linearly independent eigenfunctions $\left(\boldsymbol{v}_{0}^{ \pm}, q_{0}^{ \pm}, f_{0}^{ \pm}\right.$) are solutions of the linearized, inviscid, eigenvalue problem

$$
\begin{aligned}
& \nabla \cdot \boldsymbol{v}_{0}-0, \quad \mathrm{i} \Omega \boldsymbol{v}_{0}--\nabla q_{0} \quad \text { in } \quad \Sigma_{0}, \\
& \boldsymbol{v}_{0} \cdot \boldsymbol{e}_{z}-0, \quad f_{0}-0 \quad \text { at } z- \pm \Lambda, \\
& \boldsymbol{v}_{0} \cdot \boldsymbol{e}_{r} \quad \mathrm{i} \Omega f_{0}=q_{0}\left|f_{0}\right| f_{0,00} \mid f_{0 z z}=0 \quad \text { at } r=1,
\end{aligned}
$$

of the form

$$
\begin{aligned}
& \boldsymbol{v}_{0}^{\frac{1}{0}}-\boldsymbol{V}_{0}^{\frac{1}{0}}(r, z) \mathrm{e}^{ \pm \mathrm{i} \theta}, \quad q_{0}^{\dot{-}}-Q_{0}(r, z) \mathrm{e}^{ \pm \mathrm{i} \theta}, \\
& f_{0}^{+}-F_{00}(z) \mathrm{e}^{\perp i \theta} \text {, }
\end{aligned}
$$

where

$$
\boldsymbol{V}_{0}^{-}-V_{0} \boldsymbol{e}_{r} \pm V_{0} \boldsymbol{e}_{0}+W_{0} \boldsymbol{e}_{z},
$$

with the scalar functions $U_{0}, V_{0}, W_{0}, Q_{0}$, and $F_{0}$ such that

$$
i V_{0}, V_{0}, i W_{0}, Q_{01} \text {, and } F_{0} \text { are real. }
$$

The cigenmodes can be selected in that way because of the invariance of (24)-(26) under the symmetry

$$
\boldsymbol{v}_{0} \rightarrow-\overline{\boldsymbol{v}_{0}}, \quad q_{0} \rightarrow q_{0}, \quad f_{0} \rightarrow f_{0},
$$

which comes from invariance under arimuthal rellections. The characteristic equation providing $\Omega$ and the eigenfunctions (27) are given by Eqs. (A1) and (A2) and (A3)-(A6) in Appendix A. as taken from Ref. 25.

\section{A. Amplitude equations}

As mentioned above, only one of the amplitude equations needs to be obtained (the other one follows from symmetry considerations). For convenience we write the equation for $A$ as

$$
\begin{gathered}
\varepsilon^{3} A^{\prime}-\varepsilon C^{1 / 2} H_{1}+\varepsilon C H_{2}+(\omega-\Omega) H_{3} \\
\left|\varepsilon^{3} H_{4}\right| a H_{5} \mid \cdots .
\end{gathered}
$$

The coefficients $H_{1}, \ldots, H_{5}$ can be obtained by eliminating secular terms ${ }^{24}$ namely by imposing that all solutions of the problems giving ( $v_{k}, q_{k}, f_{k}$ ) for $k=1, \ldots, 6$ be bounded in the fast time scalc. The system of equations for those problems results from substituting (22) and (23) into the equations obtained replacing

$$
\partial / \partial t \rightarrow \partial / \partial t+\varepsilon^{2} \partial / \partial \tau
$$

into (2) and (3), and equating the coefficients of $\varepsilon \sqrt{C}, \ldots, \varepsilon^{3}, a$. The transformation (32) results from the use of two time scales. The boundary conditions are obtained by applying matching conditions to the solutions in the Stokes and the interface boundary layers. In our case, however, as well as in those which contain contact lines the simple application of this standard method may lead to incorrect results. ${ }^{26,27}$ This is so because the velocity ficld in the bulk exhibits a singularity at the rim of the disks (i.e., at $r-1, z$ $- \pm \Lambda$ ) that becomes increasingly problematic as one proceeds to higher order terms, prohibiting the integration-byparts involved in the derivation of the coefficients. We avoid this difliculty by deriving an integral solvability condition for the original problem, which has a harmless, weak singularity. This condition was first introduced in Ref. 14 for axisymmetric liquid bridges, and further used in Refs. 11, 23, and 28 . Here we extend this approach to the nonaxisymmetric case as follows. We first multiply $\boldsymbol{v}_{0}^{+}$by the equation oblaincd applying (32) to (3), and the second of Eqs. (24) (giving $\boldsymbol{v}_{0}^{+}$) by $\boldsymbol{v}$, then add and integrate in the domain

$$
\Sigma(t, \tau): 0<r<f, 0<\theta<2 \pi,-\Lambda<z<\Lambda,
$$

to oblain

$$
\begin{aligned}
& \int_{\Sigma} \boldsymbol{v}_{0} \cdot\left[\partial \boldsymbol{v} / \partial t \mid \varepsilon^{2} \partial \boldsymbol{v} / \partial \boldsymbol{j} \text { i } \Omega \boldsymbol{v} \quad \boldsymbol{v}\right. \\
& \quad \times(\nabla \times \boldsymbol{v})-C \Delta \boldsymbol{v}-a \boldsymbol{h}] d \boldsymbol{x} \\
& \quad-\quad-\int_{\Sigma}\left(\boldsymbol{v}_{0}^{+} \cdot \nabla q+\nabla q_{0}^{+} \cdot \boldsymbol{v}\right) d \boldsymbol{x}+O\left(\boldsymbol{\varepsilon} a+a^{2}\right) .
\end{aligned}
$$

Here we are using the inner product

$$
\begin{aligned}
& \boldsymbol{v}_{0} \cdot \boldsymbol{v}_{1}-u_{0} u_{1}+v_{0,0} v_{1}+w_{0} w_{1} \\
& \text { if } \boldsymbol{v}_{k}=u_{k} \boldsymbol{e}_{r}\left|v_{k} \boldsymbol{e}_{\theta}\right| w_{k} \boldsymbol{e}_{z} \text { for } k=0,1 .
\end{aligned}
$$

If Eq. (34) is multiplied by e ${ }^{i \omega t}$, integration by parts is applicd repcatcdly, and Eqs. (2), (6), (7), (24), and (26) are replaced [recall the transformation (32)]. we obtain after some algebra

$$
\begin{aligned}
& \frac{\partial}{\partial t}\left(\mathrm{e}^{-\mathrm{i} \omega t} I_{1}\right) \mid \mathrm{e}^{-\mathrm{i} \omega t}\left[I_{2} \mid \mathrm{i}(\Omega \quad \omega) I_{1}\right] \\
& \quad=\mathrm{e}^{i \omega t}\left(I_{3}\left|I_{4}\right| I_{5} \mid I_{6}\right) \mid o(a|\varepsilon| \Omega \quad \omega \mid),
\end{aligned}
$$

where

$$
\begin{aligned}
& I_{1}-\int_{\Sigma}\left(\boldsymbol{v}_{i j}^{-} \cdot \boldsymbol{v}\right) d \boldsymbol{x}+\int_{0}^{2 \pi} \int_{-\Lambda}^{\Lambda}\left[\overline{q_{i}}(f-1) f\right]_{i=f} d \theta d z, \\
& I_{2}-\varepsilon^{2} \int_{0}^{2 \pi} \int_{-\Lambda}^{z 1}\left[\overline{q_{0}^{+}} f_{f} f-\mathrm{i} \Omega\left(1 \overline{q_{0}^{+}} \boldsymbol{v}_{\tau} \cdot N\right]_{r=f} d \theta d z,\right. \\
& I_{3}=2 C \int_{0}^{2 \pi} \int_{-\Lambda}^{\Lambda}\left[\left(\mathcal{T}_{0}^{+} \cdot \boldsymbol{v}\right) \cdot N\right. \\
& \left.(\boldsymbol{T} \cdot \boldsymbol{n}) \cdot \boldsymbol{n}\left(\boldsymbol{v}_{0 j}^{+} \cdot\left(\begin{array}{ll}
N & \boldsymbol{e}_{i}
\end{array}\right)\right)\right]_{r=f} d \theta d z \\
& \text { | } 2 C \int_{0}^{2 \pi} \int_{0}^{1}\left[\left[\boldsymbol{v}_{0}^{\prime} \cdot\left(\boldsymbol{T} \cdot \boldsymbol{e}_{z}\right)\right]_{z-\lambda}\right. \\
& \left.-\left[\boldsymbol{v}_{0}^{\prime} \cdot\left(\mathcal{T} \cdot \boldsymbol{e}_{z}\right)\right]_{z-}{ }_{\Lambda}\right] \mathrm{rdr} d \theta
\end{aligned}
$$




$$
\begin{aligned}
& I_{4}-\int_{0}^{2 \pi} \int_{-\Lambda}^{\Lambda}\left[\left(\boldsymbol{v}_{0}^{+} \cdot \boldsymbol{v}\right) f f_{t}+\boldsymbol{v}_{0}^{+} \cdot\left(f \boldsymbol{e}_{r}-N\right)(q-1)\right. \\
& \text { I }\left(f \quad \text { l) } f_{L}\left(\overline{q_{0}} f \text { । } \overline{q_{0}}\right) \quad i \Omega \overrightarrow{q_{0}}(f \quad \text { l }) f\right]_{r-f} d \theta d z \\
& +\mathrm{i} \Omega \int_{0}^{2 \pi} \int_{-\Lambda}^{\Lambda}\left[|\boldsymbol{v}|^{2} / 2-2 M\right. \\
& \text { I } \left.\left(\begin{array}{ll}
q & 1
\end{array}\right)(f \quad 1)\right]_{r=f} \overline{f_{0}} d \theta d z \\
& I_{5}=\int_{\Sigma} \boldsymbol{v}_{0}^{\prime} \cdot[\boldsymbol{v} \times(\nabla \times \boldsymbol{v})] d \boldsymbol{x} \\
& I_{6}=\mathrm{i} a \Omega^{-1}\left[\int _ { 0 } ^ { 2 \pi } \int _ { 0 } ^ { 1 } \left(\left[\overline{q_{0}} \boldsymbol{h} \cdot \boldsymbol{e}_{z}\right]_{z=\Lambda}\right.\right. \\
& \left.-\left[\overline{q_{0}^{+}} \boldsymbol{h} \cdot \boldsymbol{e}_{z}\right]_{z--\Lambda}\right) r d r d \theta \\
& \left.-\int_{0}^{2 \pi} \int_{\Lambda}^{\Lambda}\left[q_{0}^{1} \boldsymbol{h} \cdot N\right]_{r-j} d \theta d z\right]
\end{aligned}
$$

Here as above $M$ is the mean curvature of the interface, $\mathcal{T}$ and $\mathcal{T}_{0}^{ \pm}$are the rate of strain tensors associated with the velocity fields $\boldsymbol{v}$ and $\boldsymbol{v}_{0}^{ \pm}$, and

$$
N-f \boldsymbol{e}_{r}-(\partial f / \partial \theta) \boldsymbol{e}_{0}-f(\partial f / \partial z) \boldsymbol{e}_{z}-|\boldsymbol{N}| \boldsymbol{n} .
$$

Secular terms are eliminated by integrating (36) over the interval $[0, t]$, dividing by $t$ and lelling $t \rightarrow \infty$. In this manner, we obtain

$$
\begin{aligned}
& \lim t^{-1} \int_{0}^{t} \mathrm{e}^{-\mathrm{i} \omega t}\left[I_{2}+\mathrm{i}(\Omega-\omega) I_{1}-I_{3}-I_{4}-I_{5}-I_{6}\right] d t-0 \\
& \quad \text { as } t \rightarrow \infty
\end{aligned}
$$

which can also be written as

resonant part of

$$
I_{2}+\mathrm{i}(\Omega-\omega) I_{1}-\left(I_{3}+I_{4}+I_{5}+I_{6}\right)-0
$$

where resonamt part refers to the terms depending on $t$ as $\mathrm{e}^{\mathrm{i} \omega t}$.

The physical interpretation of the integral solvability condition (38) is as follows. $I_{2}$ leads to those terms accounting for inertia in the amplitude equations. The real part of $I_{3}$ leads to viscous dissipation in the Stokes boundary layers atlached to the disks (which is $O\left(C^{1 / 2}\right)$ because the components of the tangential strain at the disks, $\left(\mathcal{T} \cdot \boldsymbol{e}_{z}\right) \cdot \boldsymbol{e}_{i}$ and $\left(\mathcal{T} \cdot e_{z}\right) \cdot e_{\ell}$, are of the order of $\left.1 / C^{1 / 2}\right)$, and in the bulk [which is $O(C)$ ]. The imaginary part of $I_{3}$ and $\mathrm{i}(\omega-\Omega) I_{1}$ provide the effect of detuning. $I_{4}$ and a part of $I_{5}$ yield the Stokes cubic nonlinearity, the remaining part of $I_{5}$ leads to the effect of the streaming flow and $I_{6}$ provides the direct forcing term. Note that inertia, viscous dissipation, nonlinearity and forcing, each one involves volumetric effects that have been reduced to surface integrals (along the disks and the free surface) by (repeated) integration-by-parts, which can be used withoul any danger of wrong results because the integral solvability condition is directly obtained from the original unperturbed problem (2), (3), (6), (7) and the linear problem (24)-(26), and these two problems cxhibit only a weak singularity. Also, since both the disks and the free surface are within the boundary layers attached to them, the velocity $v$ and the stagnation pressure $q$ appcaring in the surface integrals must be taken from the solutions in the boundary layers; $\boldsymbol{v}_{0}^{+}$and $q_{0}^{+}$, by contrast, are given by the lincar problem (24)-(26).

\section{Derivation of the terms $H_{1}, \ldots, H_{5}$ in Eq. (31)}

In the amplitude equation (31), $\mathrm{H}_{1}$ and $\mathrm{H}_{2}$ are associated with a strictly linear effect, allowing us to use the results of the linear analysis in Ref. 11 [also based on an integral solvability condition that is equivalent to the linearization of (38)], to oblain

$$
H_{1}--(1+\mathrm{i}) \alpha_{1} A, \quad H_{2}-\alpha_{2} A,
$$

where the real coefficients $\alpha_{1}$ and $\alpha_{2}$ are plotted in Fig. 2, as taken from Ref. 11. Note that $\alpha_{2} / \alpha_{1}$ is fairly large, as anticipated above.

The terms $H_{3}$ and $H_{4}$ are obtained by substituting Eqs. (22) - (23) and (31) into the integral solvability condition (38) and setting to zero in the resulting equation the coefficients of $O(|\omega \Omega|)$ and $O\left(\varepsilon^{3}\right)$, respectively. This yields

$$
\begin{aligned}
& I_{3}-\mathrm{i}, \quad / I_{4}-\mathrm{i} \alpha_{3}|A|^{2} A+\mathrm{i} \alpha_{4}|\beta|^{2} A \\
& -\mathrm{i} \alpha_{5} A \int_{\Lambda}^{\Lambda} \int_{0}^{2 \pi} \int_{0}^{1}\left(\boldsymbol{g} \cdot \boldsymbol{v}_{2}^{s}\right) r d r d \theta d z
\end{aligned}
$$

where the real vector $g$ is given by

$$
\boldsymbol{g}(r, z)-\mathrm{i} \Omega \Omega^{-1} \nabla \times\left(\overline{\boldsymbol{V}_{0}^{+}} \times \boldsymbol{V}_{0}^{+}\right)-g\left(r_{-} z\right) \boldsymbol{e}_{\theta},
$$

with

$$
g(r, z)-2 \mathrm{i} \Omega^{-1}\left[\partial\left(U_{0} V_{0}\right) / \partial r+\partial\left(W_{0} V_{0}\right) / \partial z\right]
$$

An illustration of this function is shown in Fig. 3. The real coefficients $\alpha_{3}, \alpha_{4}$, and $\alpha_{5}$ are given by

$$
\begin{aligned}
& \alpha_{3} \int_{-\Lambda}^{\Lambda} F_{0}(z) Q_{0}(1, z) d z-\frac{\Omega}{2} \int_{-\Lambda}^{\Lambda}\left[\left[\Omega^{2} F_{00}+3\left(\Omega^{2}+2\right) F_{22}\right] F_{0}^{2}-\left(F_{010}^{\prime}+F_{22}^{\prime}\right) F_{0} F_{0}^{\prime}+\left[2\left(V_{0} V_{22}-W_{0} W_{22}\right)+\left(F_{22}+F_{00}\right)\right.\right. \\
& \left.\left.\times\left(\begin{array}{ll}
V_{0}^{2} & Q_{0}
\end{array}\right) \mid\left(\begin{array}{ll}
F_{22} & F_{00}
\end{array}\right) W_{0}^{2}\right]_{r=1} F_{0}\right] d z \mid \frac{\Omega}{4} \int_{-\Lambda}^{\Lambda}\left[\left(F_{0} \mid 17 F_{0}^{\prime \prime}\right) F_{0}^{\prime 2} \mid 4 F_{0} F_{0}^{\prime 2} \quad\left(\Omega^{2} \quad 6\right) F_{0}^{3}\right. \\
& \left.\left[W_{0}^{2}+9 V_{i}^{2} \quad 12 i V_{0} U_{0}\right]_{i=1}\right] F_{0} d z
\end{aligned}
$$



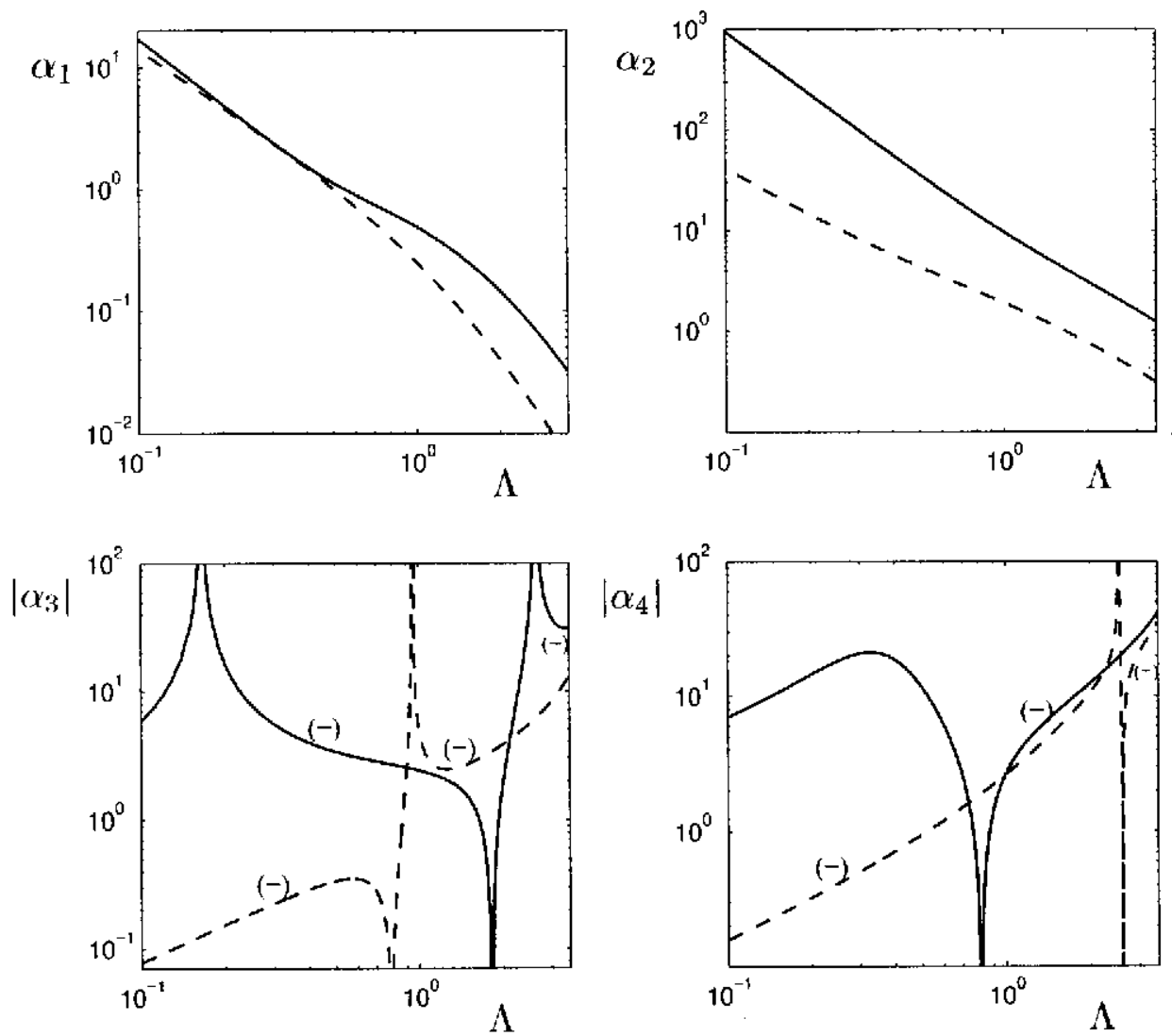

FIG. 2. The coefficients $\alpha_{1}, \ldots, \alpha_{5}$ of the amplitude equations (12) and (13), and the contributions to $\alpha_{6} \mid \mathrm{sec}$ Fiq. (47) $\mid, \alpha_{6}^{b}$ and $\alpha_{65}^{t} ; \alpha_{1}, \alpha_{2}, \alpha_{5}$ and $\alpha_{6}^{l}$ are always positive, and the branches, where $\alpha_{3}, \alpha_{4}$, and $\alpha_{6}^{b}$ are negative, are indicated. Dashed and solid lines correspond, respectively, to the capillary modes with $n \quad 0$ and 1 , where $n$ is the axial wave number (i.e., the number of nodal planes parallel to the disks).
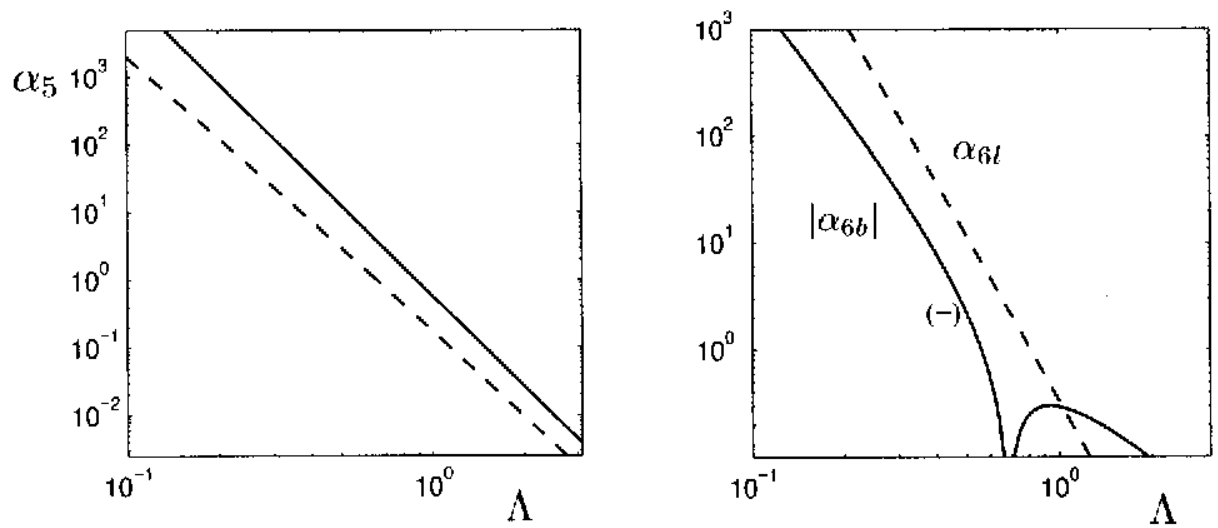

$$
\begin{aligned}
& \alpha_{4} \int_{-\Lambda}^{\Lambda} F_{0}(z) Q_{0}(1, z) d z--\frac{\Omega}{2} \int_{-\Lambda}^{\Lambda}\left[\left[\Omega^{2} F_{00}+\left(\Omega^{2}+6\right) F_{02}+3 \Omega^{2} F_{20}\right] F_{0}^{2}+\left(F_{00}^{\prime}+F_{20}^{\prime}+F_{02}^{\prime}\right) F_{00} F_{0}^{\prime}\right. \\
& \text { I } \left.\left[2 W_{0} W_{20} \mid\left(F_{00}\left|F_{20}\right| F_{02}\right) Q_{0}\right]_{i=1} F_{0}\right] d z \quad \frac{\Omega}{2} \int_{-\Lambda}^{\Lambda}\left[\left(F_{00} \quad F_{20} \mid F_{02}\right) W_{0}^{2}\right. \\
& \left.-\left(F_{000}-F_{20}-F_{02}\right) V_{0}^{2}\right]_{F-1} d z+\frac{\Omega}{2} \int_{\Lambda}^{\Lambda}\left[\left(F_{0}+17 F_{0}^{\prime \prime}\right) F_{0}^{\prime 2}+4 F_{0}^{\prime \prime 2} F_{0}^{\prime}+\left(\Omega^{2}-6\right) F_{0}^{3}\right. \\
& \left.-\left[W_{0}^{2}-3 V_{0}^{2}+4 \mathrm{i} V_{0} V_{0}\right]_{r-1}\right] F_{0} d z \\
& \alpha_{5} \int_{-\Lambda}^{\Lambda} F_{0}(z) Q_{0}(1, z) d z-\frac{\Omega}{4 \pi} \text {. }
\end{aligned}
$$

Here, the functions $V_{k l}, W_{k l}, Q_{k l}$, and $F_{k l}$, for $(k, l)=(0,0),(2,0),(0,2)$, and $(2,2)$ come from the $O\left(\varepsilon^{2}\right)$ terms in the expansions (22) and (23), see Appendix B. In order to obtain Eq. (40) we have used the fact that $\left[\right.$ since $\nabla \times \boldsymbol{v}_{0}^{-}=\mathbf{0}$, see Eq. (24)] the contribution of $I_{5}$ to the solvability condition (38) at order $\varepsilon^{3}$ is given by [recall that we are using the inner product (35)] 

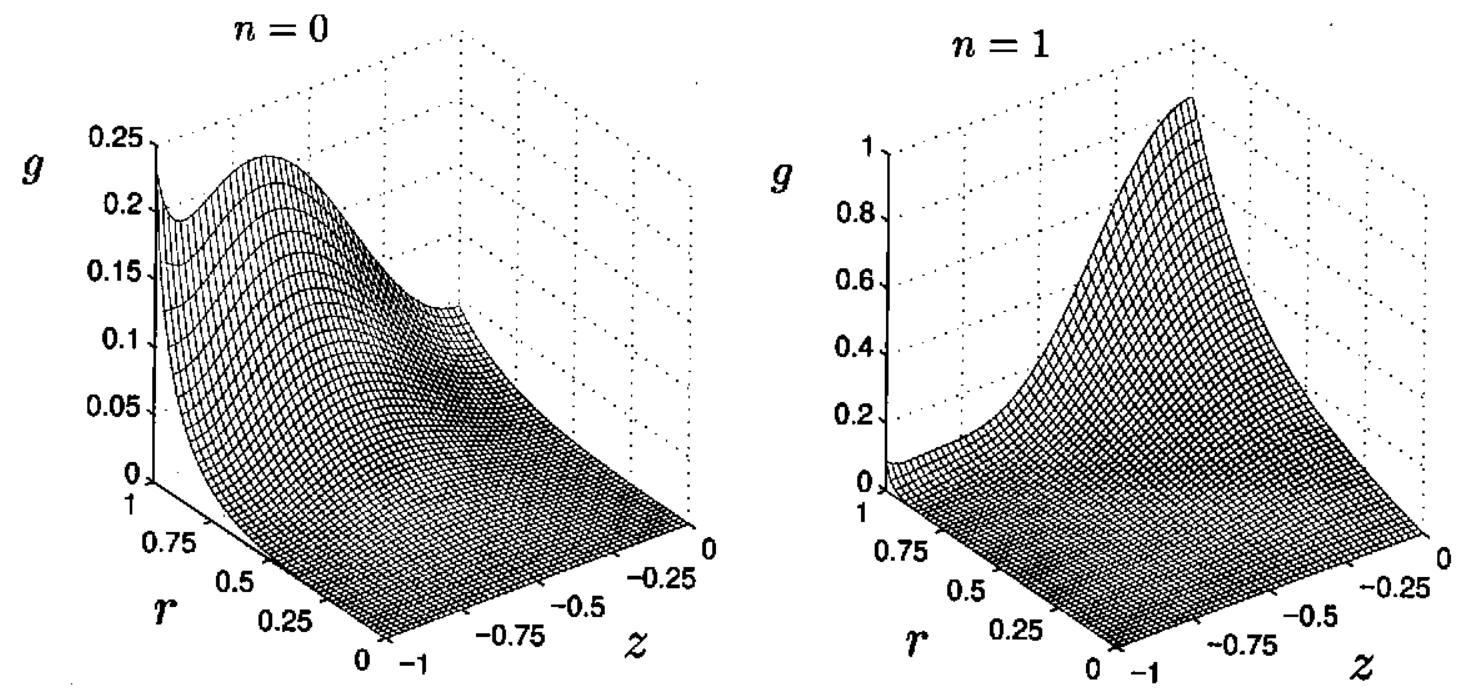

FIG. 3. The scalar function $g$ appearing in the definition (41) of $g$ for $\Lambda \quad 1$ and the indicated values of $n$. The function $g$ is even in the $z$ variable, and only negative values of $z$ are plotted.

$$
\begin{gathered}
\int_{\Sigma_{0}} \boldsymbol{v}_{0}^{+} \cdot\left(\boldsymbol{v}_{0}^{+} \times\left(\nabla \times \boldsymbol{v}_{2}^{s}\right)\right) d \boldsymbol{x}-\int_{\Sigma_{0}}\left(\nabla \times \boldsymbol{v}_{2}^{s}\right) \cdot\left(\overline{\boldsymbol{v}_{0}^{+}} \times \boldsymbol{v}_{0 j}^{+}\right) d \boldsymbol{x}-\int_{\Sigma_{0}}\left[\boldsymbol{v}_{2}^{s} \cdot\left(\nabla \times\left(\overline{\boldsymbol{v}_{0}} \times \boldsymbol{v}_{0}^{+}\right)\right)+\nabla \cdot\left(\left(\boldsymbol{v}_{2}^{s} \cdot \boldsymbol{v}_{0}^{+}\right) \overline{\boldsymbol{v}_{0}^{+}}-\text {c.c. }\right)\right] d \boldsymbol{x} \\
-\int_{\Sigma_{0}} \boldsymbol{v}_{2}^{s} \cdot\left(\nabla \times\left(\overline{\boldsymbol{V}_{0}^{+}} \times \boldsymbol{V}_{0}^{+}\right)\right) d \boldsymbol{x}+\int_{0}^{2 \pi} \int_{-\Lambda}^{\Lambda}\left[\left(\boldsymbol{v}_{2}^{s} \cdot \boldsymbol{V}_{0}^{+}\right)\left(\boldsymbol{c}_{r} \cdot \overline{\boldsymbol{V}_{0}^{+}}\right)-\text {c.c. }\right]_{i-1} d \theta d z,
\end{gathered}
$$

where we have taken into account the boundary condition (25) and the expressions (27) and (28) for $v_{0}^{\frac{1}{0}}$ and $V_{0}^{\perp}$. The first term in the right hand side is [cf. (41)] $-\mathrm{i} \Omega \int_{\Sigma_{0}} g$ $\boldsymbol{v}_{2}^{s} d \boldsymbol{x}$ and provides the efrect of the streaming flow on the amplitude equation [i.e., the last term in the right hand side of (40)]; the second term in the right hand side of (46) cancels, in Eq. (38), with other terms coming from $I_{4}$. The real coefficients $\alpha_{3}, \alpha_{4}$, and $\alpha_{5}$ are plotted in Fig. 2. Observe that the coefficients associated with the cubic nonlinearity, $\alpha_{3}$ and $\alpha_{4}$, change their signs both by crossing zeroes and through asymptotes. The latter appear at second-order internal resonances (i.e., when either $2 \Omega$ or 0 is also an eigenfrequency), which have been excluded from the analysis.

Finally, the term $\mathrm{H}_{5}$ is obtained in straightforward manner by substituting (22)-(23) and (31) into the solvability condition (38) and requiring the $O(a)$ coefficient to vanish. The result obtained may be written as

$$
\begin{aligned}
& I_{5}-\mathrm{i} \alpha_{6}, \\
& \text { with } \quad \alpha_{6}-\mu \alpha_{6}^{b}+(1-\mu) \alpha_{6}^{l} .
\end{aligned}
$$

where $\alpha_{6}^{b}$ and $\alpha_{6}^{l}$ are (real and) given by

$$
\begin{aligned}
& \alpha_{6}^{b} \int_{-\Lambda}^{\Lambda} F_{0}(z) Q_{0}(1, z) d z \\
& =\frac{\Omega}{2 \pi} \int_{0}^{2 \pi} \int_{-\Lambda}^{\Lambda} Q_{0}(1, z) \mathrm{e}^{\mathrm{i} \theta} \cos \theta z d \theta d z \\
& \quad \quad \frac{\Omega}{2 \pi} \int_{0}^{2 \pi} \int_{0}^{1}\left[Q_{0}(r, \Lambda) \quad Q_{0}(r, \quad \Lambda)\right] \mathrm{e}^{-\mathrm{i} \theta} \\
& \quad \times \cos \theta r^{2} d r d \theta \\
& \alpha_{6}^{l} \int_{-\Lambda}^{\Lambda} F_{0}(z) Q_{0}(1, z) d z \\
& \quad-\frac{\Omega}{2 \pi} \int_{0}^{2 \pi} \int_{\Lambda}^{\Lambda} Q_{0}(1, z) \mathrm{c}^{-\mathrm{i} \theta} \cos \theta d \theta d z .
\end{aligned}
$$

These are plotted in Fig. 2. Note that $\alpha_{6}^{b}-0$ and $\alpha_{6}^{l} \neq 0$ if $Q_{0}$ is an even function of $z$, and that the opposite is true if $Q_{0}$ is an odd function.

Combining the above results in Eqs. (39) and (40) and (47), and utilizing the symmetry of the problem under azimuthal rellection (20), we obtain the cvolution equations (12) and (13) for the complex amplitudes of the two counterrotating waves.

\section{B. Streaming flow equations}

The evolution equations for $\boldsymbol{v}_{2}^{s}$ are obtained from the short-time-average of the continuity cquation (2) at order $\varepsilon^{2}$, i.e., 


$$
\nabla \cdot \boldsymbol{v}_{2}^{s}-0
$$

and of the momentum equation (3) at order $\varepsilon^{4}$. The latter involves (in the quadratic, convective term) products of $O(\varepsilon)$ and $O\left(\varepsilon^{3}\right)$ terms. The solution at order $\varepsilon^{3}$ in the expansions (22) and (23), $\left(\boldsymbol{v}_{5}, q_{5}, f_{5}\right)$, may be written as

$$
\left(\boldsymbol{v}_{5}, q_{5}, f_{5}\right)-\mathrm{e}^{\mathrm{i} \omega t}\left(\boldsymbol{v}_{51}, q_{51}, f_{51}\right)+\text { c.c. } .
$$

where nonresonant terms are omitted. Using Eqs. (3), (22), (23), and (32), $v_{51}$ is seen to satisfy the following equation (recall that $|\omega-\Omega| \ll 1$ ):

$$
\begin{aligned}
& \mathrm{i} \Omega \boldsymbol{v}_{51}+\boldsymbol{v}_{0}^{+} d A / d \tau+\boldsymbol{v}_{0}^{-} d B / d \tau-\left(A \boldsymbol{v}_{01}^{-}+B \boldsymbol{v}_{0}^{-}\right) \\
& \quad \times\left(\nabla \times \boldsymbol{v}_{2}^{s}\right)=\nabla q_{51},
\end{aligned}
$$

where, since $\nabla \times \boldsymbol{v}_{0}^{-}=\mathbf{0}$, we have that

$$
\nabla \times \boldsymbol{v}_{51}=\text { i } \Omega{ }^{1} \nabla \times\left[\left(A \boldsymbol{v}_{0}^{+} \mid B \boldsymbol{v}_{0}^{-}\right) \times\left(\nabla \times \boldsymbol{v}_{2}^{s}\right)\right] .
$$

Invoking (22), (23), (50), and (52) the short-lime average of (3) at order $\varepsilon^{4}$ yields

$$
\begin{aligned}
\boldsymbol{v}_{2 \tau}^{s} & -\boldsymbol{v}_{2}^{s} \times\left(\nabla \times \boldsymbol{v}_{2}^{s}\right)-\left[\left(A \boldsymbol{v}_{0}^{+}+B \boldsymbol{v}_{0}^{-}\right) \times\left(\nabla \times \overline{\boldsymbol{v}_{51}}\right)+\text { c.c. }\right] \\
& --\nabla q_{70}+\left(C / \varepsilon^{2}\right) \Delta \boldsymbol{v}_{2}^{s},
\end{aligned}
$$

where $q_{70}$ is the $O\left(\varepsilon^{4}\right)$ term in the expansion for the shortlime-averaged stagnation pressure. Substituting (52) into (53) and using formula (A7) in Appendix A (with $\boldsymbol{u}-A \boldsymbol{v}_{0}^{+}$ $+B \boldsymbol{v}_{0}^{-}$and $\left.\boldsymbol{w}-\boldsymbol{v}_{2}^{s}\right)$ we obtain

$$
\begin{gathered}
\boldsymbol{v}_{2 \tau}^{s}-\left[\boldsymbol{v}_{2}^{s}+\left(|\beta|^{2}-|A|^{2}\right) \boldsymbol{g}\right] \times\left(\nabla \times \boldsymbol{v}_{2}^{s}\right) \\
--\nabla \widetilde{q}^{s}+\left(C / \varepsilon^{2}\right) \Delta \boldsymbol{v}_{2}^{s},
\end{gathered}
$$

where $g$ is defined by (41), and the modified pressure $\widetilde{q}^{s}$ is given by

$$
\widetilde{q}^{s}-q_{70}-\mathrm{i} \Omega^{-1}\left(|\beta|^{2}-|A|^{2}\right)\left[\left(\nabla \times \boldsymbol{v}_{2}^{s}\right) \cdot\left(\overline{V_{0}} \times V_{0}^{s}\right)\right] .
$$

The momentum equation (54) could be obtained by keeping the leading order effects of inertia (including convectionj, pressure gradients, and viscous diffusion of the streaming flow, which are the only ingredients of the original momentum equation (3) that can contribute to that flow, regardless of their orders of magnilude. Nevertheless, Eq. (54) has been more easily derived by assuming that $C / \varepsilon^{2}$ is order unity.

The appropriate boundary conditions for Eqs. (49) and (54) are obtained by applying matching conditions to the slowly varying part of the solution in the boundary layers attached to the disks and the free surface at orders $\varepsilon^{2}$ and $\varepsilon^{2} C^{1 / 2}$. respectively. For the sake of brevity we do not give here this analysis, which can be found in the doctoral thesis of the first author of this paper, which is available upon request. A direct application of these results yields

$$
\begin{aligned}
& \boldsymbol{v}_{2}^{s}=\left(\left.|A|^{2}|| B\right|^{2}\right) \varphi_{1} \boldsymbol{e}_{r}
\end{aligned}
$$

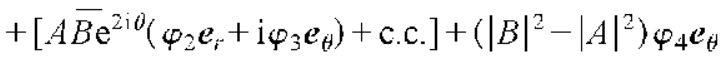

$$
\begin{aligned}
& \text { at } z- \pm \Lambda \text {, } \\
& \boldsymbol{v}_{2}^{s} \cdot \boldsymbol{e}_{r}=0, \quad\left(\boldsymbol{v}_{2,}^{*}, \quad \boldsymbol{v}_{2}^{*}\right) \cdot \boldsymbol{e}_{\emptyset}=\left(|B|^{2} \quad|A|^{2}\right) \varphi_{\varsigma}, \\
& \boldsymbol{v}_{2 r}^{s} \cdot \boldsymbol{e}_{z}-0 \text { at } r-1 \text {, }
\end{aligned}
$$

where the real functions $\varphi_{1}, \ldots, \varphi_{4}$ and $\varphi_{5}$ are

$$
\begin{aligned}
& \varphi_{1}(r)=\Omega^{1}\left(U_{0 r} U_{0} \quad V_{0 r} V_{0} \quad 2 U_{00} W_{0 z}\right)_{z=+\Lambda}, \\
& \varphi_{2}(r)-\Omega^{-1}\left(U_{0,} U_{0}+V_{0 r} V_{0}-2 U_{0} W_{0 z}\right)_{z-1}, \\
& \varphi_{3}(r)=\Omega^{-1}\left(2 \mathrm{i} V_{0} W_{0 z} \mid r^{-1}\left(U_{0}^{2} \mid V_{0}^{2}\right)\right)_{z=+\Lambda}, \\
& \varphi_{4}(r)-3 \mathrm{i} \Omega{ }^{1}\left(V_{0} W_{0 z}\right)_{z=+\Lambda} \\
& \varphi_{5}(z)--4 \Omega^{-1}\left(U_{0} U_{0 r}+W_{0} U_{0 z}+W_{0}^{2}+\mathrm{i} V_{0} U_{0 r}\right)_{r-1} .
\end{aligned}
$$

These scalar functions are plotted in Fig. 4 for the first two modes. The functions $\varphi_{3}, \varphi_{4}$, and $\varphi_{5}$ diverge at the rim of the disks, but this singularity is logarithmic and does not present major difficulty. Note that $\varphi_{5}$ is larger than $\varphi_{1}, \ldots$, and $\varphi_{4}$, especially for $n=1$ and for higher-order modes not shown here. This is because $\varphi_{1}, \ldots$, and $\varphi_{4}$ account for the forcing effect of the Stokes boundary layers attached to the disks, which are much weaker than the interface boundary layer responsible for $\varphi_{5}$. By the word weaker we mean that the variation of the oscillatory velocity through the Stokes boundary layers is small compared with typical velocities in the bridge [this was also the reason for the dominance of $\alpha_{2}$ over $\alpha_{1}$ in the damping rate (18)], while the variation of the oscillatory tangential stress through the interface boundary layer is comparable to the characteristic gradients of velocities in the bridge. The dominance of $\varphi_{5}$ will prove essential in Sec. III in obtaining a simplified model, which is more amenable to analytical and numerical treatment.

\section{ANALYSIS OF THE SLOW EVOLUTION OF THE COUNTER-ROTATING WAVES: INTERACTION WITH THE STREAMING FLOW}

The aim of this section is to analyze the influence of the streaming flow in the dynamics of the counter-rotating waves. The CASF equations have been derived considering independent the small parameters $C, \delta, \varepsilon$, and $a$. Now they are related to each other as

$$
\begin{aligned}
& \varepsilon^{2}=C^{1 / 2} \alpha_{1} \mid C \alpha_{2}, \quad \widetilde{\delta}=\delta / \varepsilon^{2} \sim 1, \\
& \text { and } \widetilde{a}-\alpha_{6} a / \varepsilon^{3} \sim 1,
\end{aligned}
$$

to re-write the CASF cquations (12)-(16) as

$$
\begin{aligned}
& A^{\prime}--(1+\mathrm{i} \widetilde{\delta}) A+\mathrm{i}\left(\alpha_{3}|A|^{2}+\alpha_{4}|B|^{2}\right) A \\
& \quad-\mathrm{i} \alpha_{5} \int_{\Sigma_{0}} \boldsymbol{g} \cdot \boldsymbol{v} d \boldsymbol{x} \lambda+\mathrm{i} \widetilde{a}, \\
& B^{\prime}--(1+\mathrm{i} \widetilde{\delta}) B+\mathrm{i}\left(\alpha_{3}|B|^{2}+\alpha_{4}|A|^{2}\right) B \\
& \quad+\mathrm{i} \alpha_{5} \int_{\Sigma_{0}} \boldsymbol{g} \cdot \boldsymbol{v} d \boldsymbol{x} B+\mathrm{i} \widetilde{a}, \\
& \nabla \cdot \boldsymbol{v}=0, \\
& \partial \boldsymbol{v} / \partial \tau-\left[\boldsymbol{v}+\left(|B|^{2}-|A|^{2}\right) \boldsymbol{g}\right] \times(\nabla \times \boldsymbol{v}) \\
& \quad--\nabla \widetilde{q}+R_{s}{ }^{1} \Delta \boldsymbol{v} \quad \text { in } \Sigma_{i j,},
\end{aligned}
$$



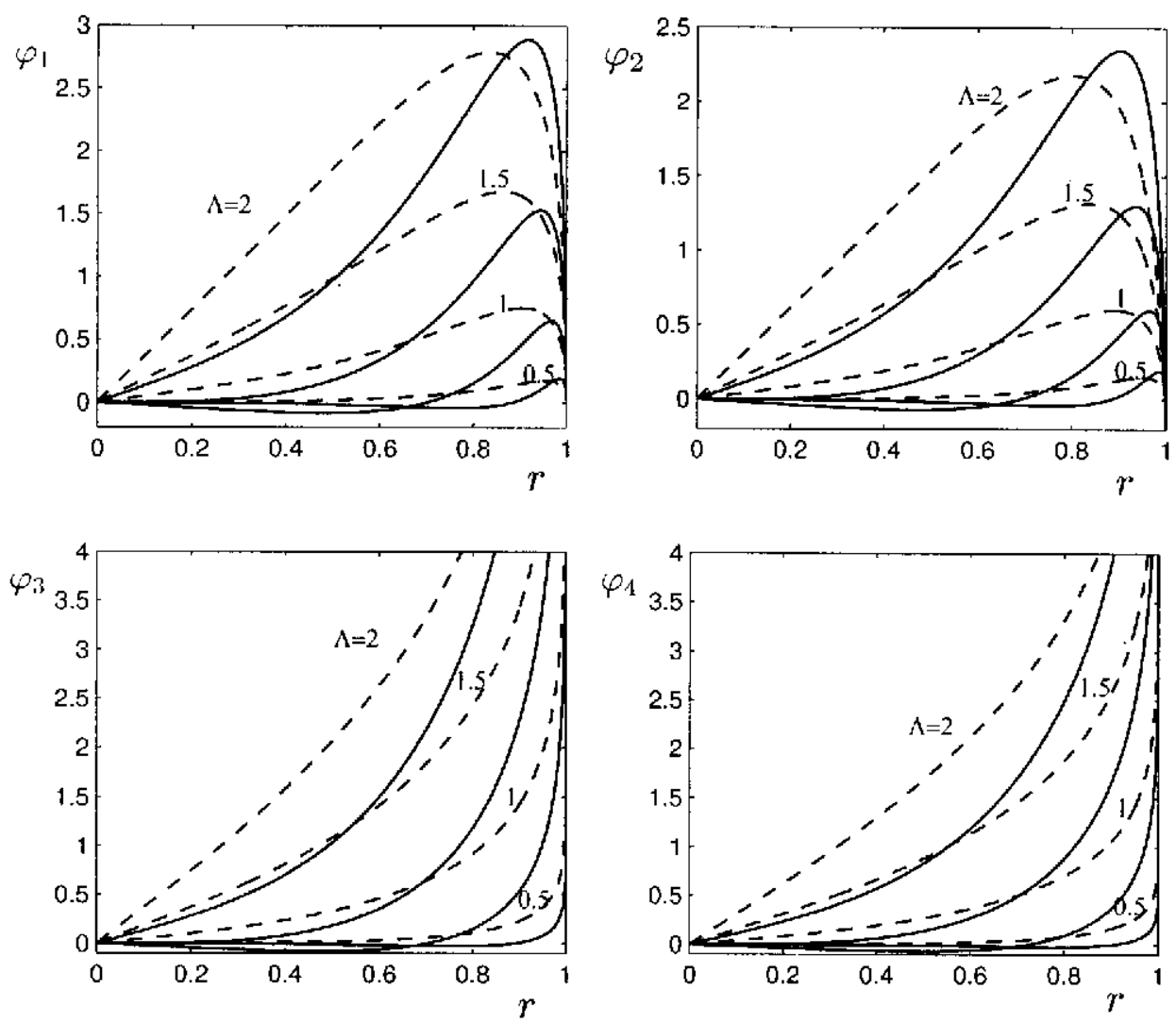

FIG. 4. The scalar functions $\varphi_{1}, \ldots$, and $\varphi_{5}$ appearing in the boundary conditions (16) and (17), as calculated from Eqs. (57), for the indicated values of $A$, and $n=0$ (dashed line) and $n=1$ (solid line).
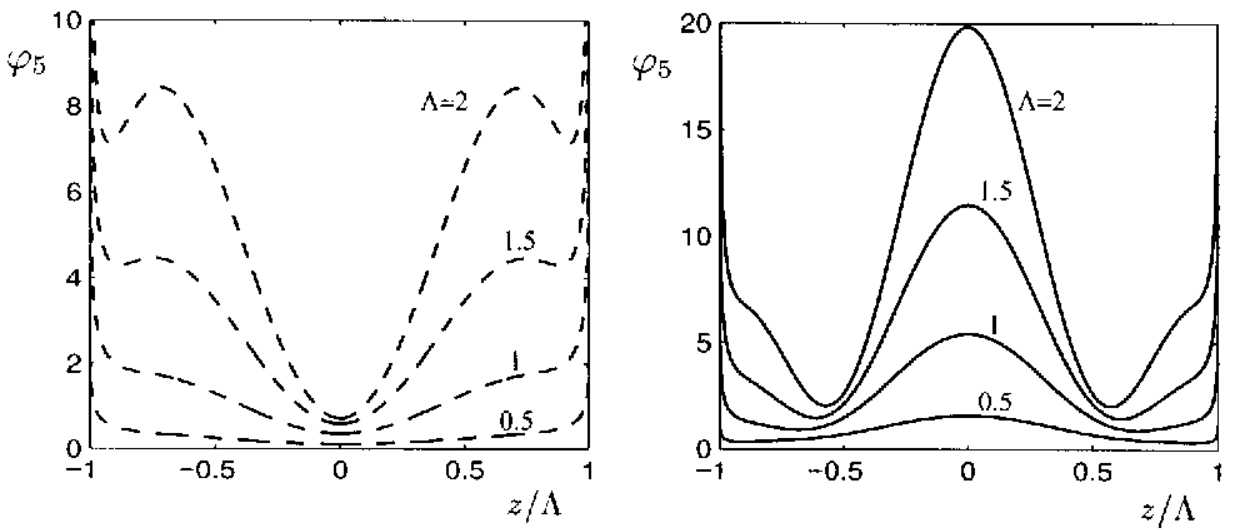

$$
\begin{aligned}
& \boldsymbol{v}=\left(\left.|A|^{2}|| B\right|^{2}\right) \varphi_{1} \boldsymbol{e}_{r} \\
&+\left[A \overline{B \mathrm{c}^{2}}{ }^{2 i \theta}\left(\varphi_{2} \boldsymbol{e}_{r}+\mathbf{i} \varphi_{3} \boldsymbol{e}_{\theta}\right)+\mathrm{c.c} .\right] \\
& \mid\left(|B|^{2} \quad|A|^{2}\right) \varphi_{4} \boldsymbol{e}_{\theta} \text { at } z= \pm \Lambda, \\
& \boldsymbol{v} \cdot \boldsymbol{e}_{r}-0, \quad(\partial \boldsymbol{v} / \partial r-\boldsymbol{v}) \cdot \boldsymbol{e}_{\theta}-\left(|B|^{2}-|A|^{2}\right) \varphi_{5},
\end{aligned}
$$$$
(\partial v / \partial r) \cdot e_{z}-0 \text { al } r-1 \text {, }
$$

where the sub and superscripts have been dropped out from $v$ and $\tilde{q}$, and the streaming fow Reynolds number $R_{s}$ is given by

$$
R_{s}-\varepsilon^{2} / C \equiv \alpha_{1} C^{-1 / 2}+\alpha_{2} .
$$

The system (59)-(64) depends on the real cocfficients $\alpha_{3}$, $\alpha_{4}, \alpha_{5}$, and $R_{s}$, and on the real functions $g . \varphi_{1}, \ldots$, and $\varphi_{5}$; all these are fixed once the liquid and the geometry are selected. The detuning parameter, $\widetilde{\delta}$, and the re-scaled forc- ing amplitude, $\tilde{a}$, instead can be varied independently through the forcing frequency and amplitude.

As in the original problem (2), (3), (6)-(8), Eqs. (59)(64) are invariant under the azimuthal reflection (20). Now, the amplitude equations (59) and (60) are decoupled from the streaming flow only if

$$
\int_{\Sigma_{0}} g \cdot v d x-0 \text { for all } \tau>0
$$

Since $g$ is independent of $\theta$ this condition is satisfied by those solutions of (59)-(64) that are reflection symmetric. i.c., invariant under (20) for all $\tau$. These are standing wowes (SWs) such that $A=B$ satisfies $A^{\prime}=(1 \mid \mathrm{i} \widetilde{\delta}) A \mid \mathrm{i}\left(\alpha_{3}\right.$ $\left.\left.+\alpha_{4}\right)|A|^{2}\right) A+\mathrm{i} \widetilde{a}$, whose solutions converge to (rellection symmetric) steady states, which correspond to monochro- 
matic SWs. Note in addition that for sufficiently small wave steepness these are the only attractors of the system. But as $\widetilde{a}$ increases nonlinear terms can promote the breaking of rellection symmetry, and the coupling with the streaming flow cannot be ignored. Of course, any other attractor different from the SWs involves necessarily the strcaming flow as well, and their orbits cannot be reflection symmetric for all $\tau>0$, though the averaged altractor can be.

The analysis of the CASF equations beyond the simplest SWs must rely on an expensive numerical calculation unless such equations are further simplified. In Sec. III A we consider a first simplification, which results from the weakness of the Stokes boundary layers attached to the disks. The resulting equations are used to analy $/ \mathrm{e}$ the stability of the simplest monochromatic SWs, and are further simplified by either (i) neglecting convective terms in momentum equations (see Sec. III A 1), or (ii) using a Galerkin projection of the streaming flow into a set of few hydrodynamic modes (see Sec. III A 2).

\section{An approximation for weak Stokes boundary layer}

This simplification relics on the observation made above that $\left|\varphi_{1}\right| \ldots$, and $\left|\varphi_{4}\right|$ are small compared to $\left|\varphi_{5}\right|$ for the second mode with $n-1$, (sec Fig. 4). Thus, we neglect the tangential velocity at the disks and replace the boundary condition (63) by

$$
\boldsymbol{v}=\boldsymbol{0} \quad \text { at } z= \pm \Lambda .
$$

Equations (59)-(62), (64), and (67) will be referred to as approximated coupled amplitude-streaming flow (ACASF) equations in the sequel. The reflection symmetric steady states of these equations are of the form $A=B=A_{0}, \boldsymbol{v}=\mathbf{0}$, $\widetilde{q}^{-}$constant, where $A_{0}$ is given by

$$
(1+\mathrm{i} \widetilde{\delta}) A_{0}-\mathrm{i}\left(\alpha_{3}+\alpha_{4}\right)\left|A_{0}\right|^{2} A_{0}-\mathrm{i} \widetilde{a} .
$$

Their linear stability is readily analyzed by first replacing

$$
\begin{aligned}
& A=A_{0}\left|\xi_{1} \mathrm{e}^{\lambda \tau}\right| \overline{\eta_{1}} \mathrm{e}^{\bar{\lambda} \tau}, \quad B=A_{0}\left|\xi_{2} \mathrm{e}^{\lambda \tau}\right| \overline{\eta_{2}} \mathrm{e}^{\bar{\lambda} \tau}, \\
& \boldsymbol{v}-V \mathrm{e}^{\lambda t} \boldsymbol{e}_{\theta}+\text { c.c., } \quad \widetilde{q}-\widetilde{Q} \mathrm{c}^{\lambda \tau}+\text { c.c. }
\end{aligned}
$$

into (59)-(62), (64), and (67), and linearizing to obtain the following linear eigenvalue problem

$$
\begin{aligned}
& {\left[\lambda+1+\mathrm{i} \widetilde{\delta}-\mathrm{i}\left(2 \alpha_{3}+\alpha_{4}\right)\left|A_{0}\right|^{2}\right] \xi_{1}-\mathrm{i} \alpha_{3} A_{0}^{2} \eta_{1}} \\
& -\mathrm{i} \alpha_{4}\left|A_{0}\right|^{2} \xi_{2}-\mathrm{i} \alpha_{4} A_{0}^{2} \eta_{2}+\mathrm{i} \alpha_{5} A_{0} \int_{\Sigma_{0}} g V d x-0, \\
& {\left[\lambda+\mathbf{l}+\mathrm{i} \widetilde{\delta}-\mathrm{i}\left(2 \alpha_{3}+\alpha_{4}\right)\left|A_{0}\right|^{2}\right] \xi_{2}-\mathrm{i} \alpha_{3} A_{0}^{2} \eta_{2}} \\
& \quad-\mathrm{i} \alpha_{4}\left|A_{0}\right|^{2} \xi_{1}-\mathrm{i} \alpha_{4} A_{0}^{2} \eta_{1}-\mathrm{i} \alpha_{5} A_{0} \int_{\Sigma_{0}} g V d x-0,
\end{aligned}
$$

$$
\begin{aligned}
& \mathrm{i} \alpha_{3} \overline{A_{0}^{2}} \xi_{1} \mid\left[\lambda|1 \quad \mathrm{i} \widetilde{\delta}| \mathrm{i}\left(2 \alpha_{3} \mid \alpha_{4}\right)\left|A_{0}\right|^{2}\right] \eta_{1} \\
& +\mathrm{i} \alpha_{4} A_{0}^{2} \xi_{2}+\mathrm{i} \alpha_{4}\left|A_{01}\right|^{2} \eta_{2}-\mathrm{i} \alpha_{5} A_{0} \int_{\Sigma_{0}} g V d x-0,
\end{aligned}
$$

$$
\begin{aligned}
& \mathrm{i} \alpha_{3} A_{0}^{2} \xi_{2}+\left[\lambda+1-\mathrm{i} \widetilde{\delta}+\mathrm{i}\left(2 \alpha_{3}+\alpha_{4}\right)\left|A_{01}\right|^{2}\right] \eta_{2} \\
& +\mathrm{i} \alpha_{4} A_{0}^{2} \xi_{1}+\mathrm{i} \alpha_{4}\left|A_{0}\right|^{2} \eta_{1}+\mathrm{i} \alpha_{5} A_{0} \int_{\Sigma_{0}} g V d x-0, \\
& \lambda V-R_{s}^{-1}\left(\partial^{2} V / \partial r^{2}+r^{-1} \partial V / \partial r-r^{-2} V+\partial^{2} V / \partial z^{2}\right) \\
& \quad \text { in } 0<r<1,|z|<\Lambda, \\
& V-0 \quad \text { at } r-0 \quad \text { and at } z-1 \Lambda, \\
& \partial V / \partial r \quad V=\varphi_{5}\left[A _ { 0 } \left(\begin{array}{lll}
\xi_{2} & \left.\xi_{1}\right) \mid A_{0}\left(\eta_{2}\right. & \left.\left.\eta_{1}\right)\right]
\end{array}\right.\right. \\
& \text { at } r-1 .
\end{aligned}
$$

Note that $w \mathrm{c}$ are only considering cigenmodes whose streaming velocity field is axisymmetric and purely azimuthal. The additional (infinitely many) modes are unforced and associated with real and strictly negative eigenvalues, ${ }^{11,27,29}$ and need not being considered.

All nontrivial solutions of (69)-(75) fall into two classes: $\xi_{1}-\xi_{2}-\eta_{1}-\eta_{2}-0$ and $\xi_{1}+\xi_{2}-\eta_{1}+\eta_{2}-0$ corresponding to reflection symmetric and antisymmetric perturbations, respectively. The associaled dispersion relations follow from the systems of equations that are obtained upon addition and subtraction of Eqs. (69) and (70), and of Eqs. (71) and (72). For symmetric perturbations we have

$$
(\lambda \mid 1)^{2}=\left(\alpha_{3} \mid \alpha_{4}\right)^{2}\left|A_{0}\right|^{4}\left[2\left(\alpha_{3} \mid \alpha_{4}\right)\left|A_{0}\right|^{2} \quad \widetilde{\delta}\right]^{2},
$$

and for antisymmetric perturbations

$$
\begin{array}{r}
(\lambda+1)^{2}-\left(\alpha_{3}-\alpha_{4}\right)^{2}\left|A_{0}\right|^{4}-\left[2 \alpha_{3}\left|A_{0}\right|^{2}-\tilde{\delta}\right]^{2} \\
4 \mathcal{H}\left|A_{0}\right|^{2}\left[\left(\begin{array}{lll}
\left(\alpha_{3} \mid \alpha_{4}\right)\left|A_{0}\right|^{2} & \tilde{\delta}
\end{array}\right]\right.
\end{array}
$$

where

$$
\mathcal{H}-2 \pi \alpha_{5} \int_{-\Lambda}^{\lambda} \int_{0}^{1} g(r, z) H(r, z) r d r d z
$$

and the function $H$ is uniquely determined (in terms of $\lambda$ ) by the linear problem

$$
\begin{aligned}
& \lambda H=R_{s}{ }^{1}\left(\partial^{2} H / \partial r^{2} \mid r^{-1} \partial H / \partial r \quad r^{-2} H\right. \\
& \left.\qquad \mid \partial^{2} H / \partial z^{2}\right) \quad \text { in } \quad 0<r<1, \quad|z|<\Lambda, \\
& I /-0 \quad \text { at } r-0 \quad \text { and at } z- \pm \Lambda, \\
& \partial H / \partial r \quad H=\varphi_{5}(z) \quad \text { at } r=1 .
\end{aligned}
$$

The dispersion relation (76) does not involve the streaming flow, and readily shows that the destabilization is always nonoscillatory (i.c., $\lambda-0$ ). In this case, the marginal stability curve corresponds to the common solutions of the following equation:

$$
\left[2\left(\alpha_{3}+\alpha_{4}\right)\left|A_{0}\right|^{2}-\widetilde{\delta}\right]^{2}-\left(\alpha_{3}+\alpha_{4}\right)^{2}\left|A_{0}\right|^{4}+1-0,
$$

which follows by letting $\lambda-0$ in (76), and

$$
\left[\left(\alpha_{3}+\alpha_{4}\right)\left|A_{0}\right|^{2}-\widetilde{\delta}\right]^{2}\left|A_{0}\right|^{2}+\left|A_{0}\right|^{2}-|\widetilde{a}|^{2} .
$$



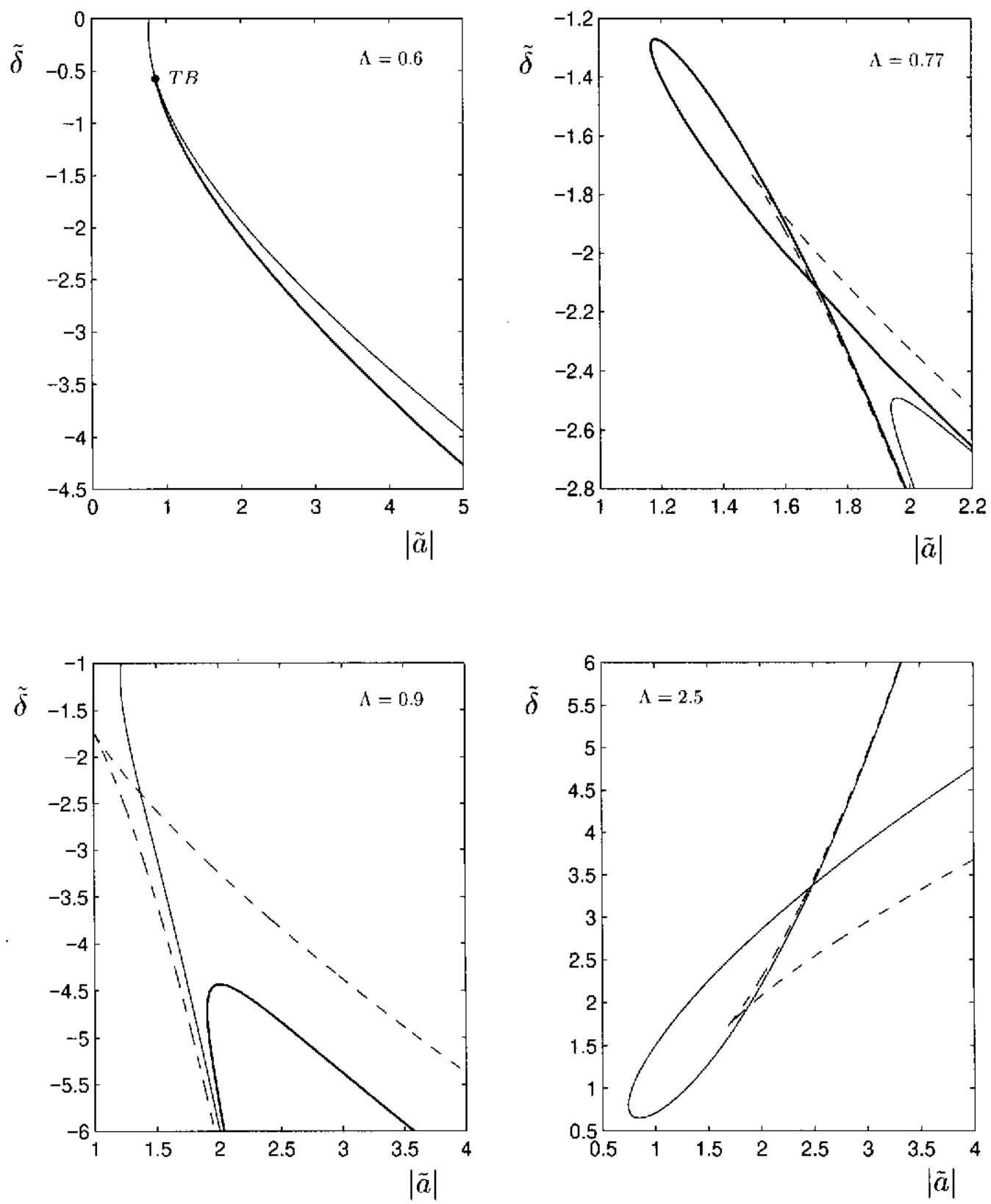

FIG. 5. Ceutral stability curves of the $\mathrm{ACASF}$ equations (59) (62), (64), and (67) for $C \quad 10^{-3}, n \quad 1$ and the indicated values of the slenderness. The numerieal values of the eocfficients $R_{s}, \alpha_{3}, \alpha_{1}$, and $\alpha_{5}$ are given in (84). The plotted eurves correspond to nonoseillatory instability under symmetric perturbations (dashed), and nonoscillatory (solid, thin) and oseillatory (solid, thick) instabilities under antisymmetric perturbations.

which in turn is obtained upon multiplication of the first equation (68) by its complex conjugate. For a fixed value of the detuning, $\widetilde{\delta}$, Eq. (82) provides a curve $\left|A_{0}\right| \quad|\widetilde{a}|$ that is cither $S$-shaped, if

$$
\left(\alpha_{3}+\alpha_{4}\right) \widetilde{\delta}>0 \text { and }|\widetilde{\delta}|>\sqrt{3} \text {, }
$$

or monotonic otherwise (see Fig. 6). In the second case Eqs. (81) and (82) have no common solutions and the unique symmetric steady state is always stable (under symmetric perturbations). If (83) holds then the intermediate branch corresponds to unstable steady states and the limiting bifurcation points are saddle-nodes. If $\left|A_{0}\right|$ is eliminated in Eqs. (81) and (82) then the resulting equation provides a cusped $|\widetilde{a}|-\widetilde{\delta}$ curve (as those plotted with dashed lines in Fig. 5). which is the common boundary of the uniqueness and multiplicity regions for symmetric steady states

The dispersion relation for antisymmetric modes (77) is richer than in the previous case. Destabilization can now occur either in a steady or oscillatory bifurcation (i.e., $\lambda$ $=0$ or $\lambda= \pm i \lambda_{I}$, with $\lambda_{I} \neq 0$ ). Note that the latter is due to the presence of the streaming flow because if we set $\mathcal{H}=0$ Eq. (77) has not purely imaginary roots. The neutral stability curves associated with these bifurcations, in the plane $|\widetilde{a}|$ $-\widetilde{\delta}$, are numerically calculated from Eqs. (77)-(80) and (82). In Fig. 5 we illustrate neutral stability curves of ACASF equations for $n=1, C=10^{3}$, and several values of the slenderness $\Lambda$. The numerical values of the coefficients $\alpha_{3}, \alpha_{4}$, $\alpha_{5}$ and $R_{s}-\alpha_{1} / \sqrt{C}+\alpha_{2}$ for these values of $\Lambda$ are 
readily obtained from Eqs. (43)-(45) and (65)

$$
\begin{aligned}
& \alpha_{3}=8.43, \quad \alpha_{4}=16.9, \quad \alpha_{5}=2.04, \\
& R_{s}-53.6 \quad \text { for } \Lambda-0.60, \\
& \alpha_{3}--4.57, \quad \alpha_{4}-1.42, \quad \alpha_{5}-1.10, \\
& R_{s}=37.1 \quad \text { for } \Lambda=0.77, \\
& \alpha_{3}--3.12, \quad \alpha_{4}--1.72, \quad \alpha_{5}-0.746, \\
& R_{s}=33.9 \quad \text { for } \Lambda=0.90, \\
& \alpha_{3}-6.52, \quad \alpha_{4}--0.460, \quad \alpha_{5}-0.0616, \\
& \quad R_{s}=4.79 \text { for } \Lambda=2.50 .
\end{aligned}
$$

The thin solid lines denote symmetry-breaking bifurcations to non-symmetric steady solutions, while the thick lines signify Hopf bifurcations producing nonsymmetric periodic solutions which retain the reflection symmetry on average. The new nonsymmetric steady solutions correspond to rotating states, where one of the counter-rotating waves always dominates. By contrast, the new periodic solutions are blinking states, in which each wave dominates over a part of the period. Note that there are some regions of Fig. 5 where both types of states can be expected.

The only bifurcation of higher codimension in Fig. 5 (and other cases we have checked) is a Takens-Bogdanov (TB), where a saddle-node and a Hopf bifurcation coalesce; the remaining intersections between neutral stability curves are simply the result of projecting onto the plane $\widetilde{a}-\widetilde{\delta}$ and do not indicate higher order degeneracies. Because the center manifold at the TB point is two-dimensional, no chaotic behavior can be conjectured from these local results (the homoclinic orbits associated with the TB point do not lead to any complexily in the absence of nonautonomous perturbations ${ }^{30}$.

In Fig. 6, also we plot several representative response curves, $\left|A_{0}\right|$ vs $\widetilde{a}$, for fixed values of the slenderness $\Lambda$ and the detuning parameter $\widetilde{\delta}$. Note that when the response curve is $S$-shaped both upper and lower branches can exhibit instability intervals, through both stationary and Hopf bifurcations.

\section{Linear approximation of the streaming flow}

Let us now neglect convective terms in Eq. (62). This approximation should give qualitatively correct results, at least for moderate forcing amplitude and/or small slenderness; see below. The momentum equation in the azimuthal direction decouples from the continuity and the remaining momentum equations, and the ACASF equations simplify to

$$
\begin{array}{r}
A^{\prime}--(1+\mathrm{i} \widetilde{\delta}) A+\mathrm{i}\left(\alpha_{3}|A|^{2}+\alpha_{4}|B|^{2}\right) A \\
2 \pi \mathrm{i} \alpha_{5} A \int_{\Lambda}^{\Lambda} \int_{0}^{1} g V r d r d z \mid \mathrm{i} \widetilde{a}, \\
B^{\prime}--(1+\mathrm{i} \widetilde{\delta}) B+\mathrm{i}\left(\alpha_{3}|B|^{2}+\alpha_{4}|A|^{2}\right) B \\
+2 \pi \mathrm{i} \alpha_{5} B \int_{-\Lambda}^{\Lambda} \int_{0}^{1} g V r d r d z+\mathrm{i} \widetilde{a}
\end{array}
$$

$$
\begin{aligned}
& \partial V / \partial \tau^{-} R \mathrm{c}^{-1}\left(\partial^{2} V / \partial r^{2}+r^{-1} \partial V / \partial r\right. \\
& \left.r^{2} V \mid \partial^{2} V / d z^{2}\right) \quad \text { in } 0<r<1, \quad|z|<\Lambda \text {, } \\
& V-0 \text { at } r-0 \text { and at } z- \pm \Lambda \text {, } \\
& \partial V / \partial r \quad V=\varphi_{5}(z)\left(|B|^{2} \quad|A|^{2}\right) \quad \text { at } r=1,
\end{aligned}
$$

where $V$ is the azimuthal velocity component. These equations also apply if the eigenfrequency $\lambda_{,}$at the Hopf bifurcation is fairly large because then, at least near the bifurcation, the convective terms can be neglected compared to $\partial \boldsymbol{v} / \partial \tau$ in the momentum equation (62). Note that both the symmetric steady states of these simplified equations and their linear stability properties are again given by $(68)$ and (69)-(75), respectively. Thus, the system (85)-(88) is cxact at this level, as compared with the ACASF equations.

Equations $(85)-(88)$ have been numerically integrated to find that the Hopf bifurcation is usually subcritical and the system jumps to a branch of nonreflection symmetric steady states that correspond to rolating waves. Thus, we have pursued (by continuation from the branch of rotating waves) periodic attractors like those shown in Fig. 7. Note that each counter-rotating wave dominates in a part of the period in Fig. 7(a), which corresponds to a blinking state, and that the wave traveling in the clockwise direction (with amplitude $|B|$ ) dominates in Fig. 7(b) (thus, this is a kind of rotating state). The system (85)-(88) exhibits other attractors like those contained in Eqs. (91), (92), (99) considered below. In fact, these equations yield results that are qualitatively similar to those obtained from Eqs. $(85)-(88)$.

\section{Three-mode Galerkin approximation of the streaming flow}

In order to get some insight into the role of (nonlinear) convective terms, not included in the simplified equation (87) we consider the projection of the streaming flow probIem (61), (62), (64) and (67) onto a manifold spanned by a few hydrodynamic modes [i.e., the eigenmodes of the lineari/ation of (61)-(64) around the quicscent state, sec Eqs. $(\mathrm{C} 2)-(\mathrm{C} 4)$ in the Appendix C]. The use of hydrodynamic modes is consistent with the fact that the streaming flow is associated with these modes, as pointed out in Sec. I. In fact, all hydrodynamic modes are retained in the continuous streaming flow equations. We shall retain only three modes: The first purcly meridional and purcly azimuthal axisy mmetric modes, and the first nonaxisymmetric mode with azimuthal wave number $m=2$. Thus, the projection of Eqs. $(61)-(64)$ onto the linear space spanned by these three modes is

$$
\begin{aligned}
& \boldsymbol{v}-Z_{1} \boldsymbol{U}_{1}+Z_{2} \boldsymbol{U}_{2}+\left(Z_{3} \boldsymbol{U}_{3}+\text { c.c. }\right), \\
& \hat{q}=Z_{1} \hat{Q}_{1}\left|Z_{2} \hat{Q}_{2}\right|\left(Z_{3} \hat{Q}_{3} \mid \text { c.c. }\right),
\end{aligned}
$$

where $\left(\boldsymbol{U}_{1}, \hat{Q}_{1}\right),\left(\boldsymbol{U}_{2}, \hat{Q}_{2}\right)$ and $\left(\boldsymbol{U}_{3}, \hat{Q}_{3}\right) \mathrm{c}^{-2 \mathrm{i} \theta}$ depend only on $r$ and $z$, and

$$
\begin{aligned}
& \boldsymbol{U}_{1}=U_{1} \boldsymbol{e}_{r} \mid W_{1} \boldsymbol{e}_{z}, \quad \boldsymbol{U}_{2}=V_{2} \boldsymbol{e}_{\theta}, \\
& \boldsymbol{U}_{3}-\left(U_{3} \boldsymbol{e}_{r}+\mathrm{i} V_{3} \boldsymbol{e}_{\theta}+W_{3} \boldsymbol{e}_{z}\right) \mathrm{e}^{2 i \theta} .
\end{aligned}
$$



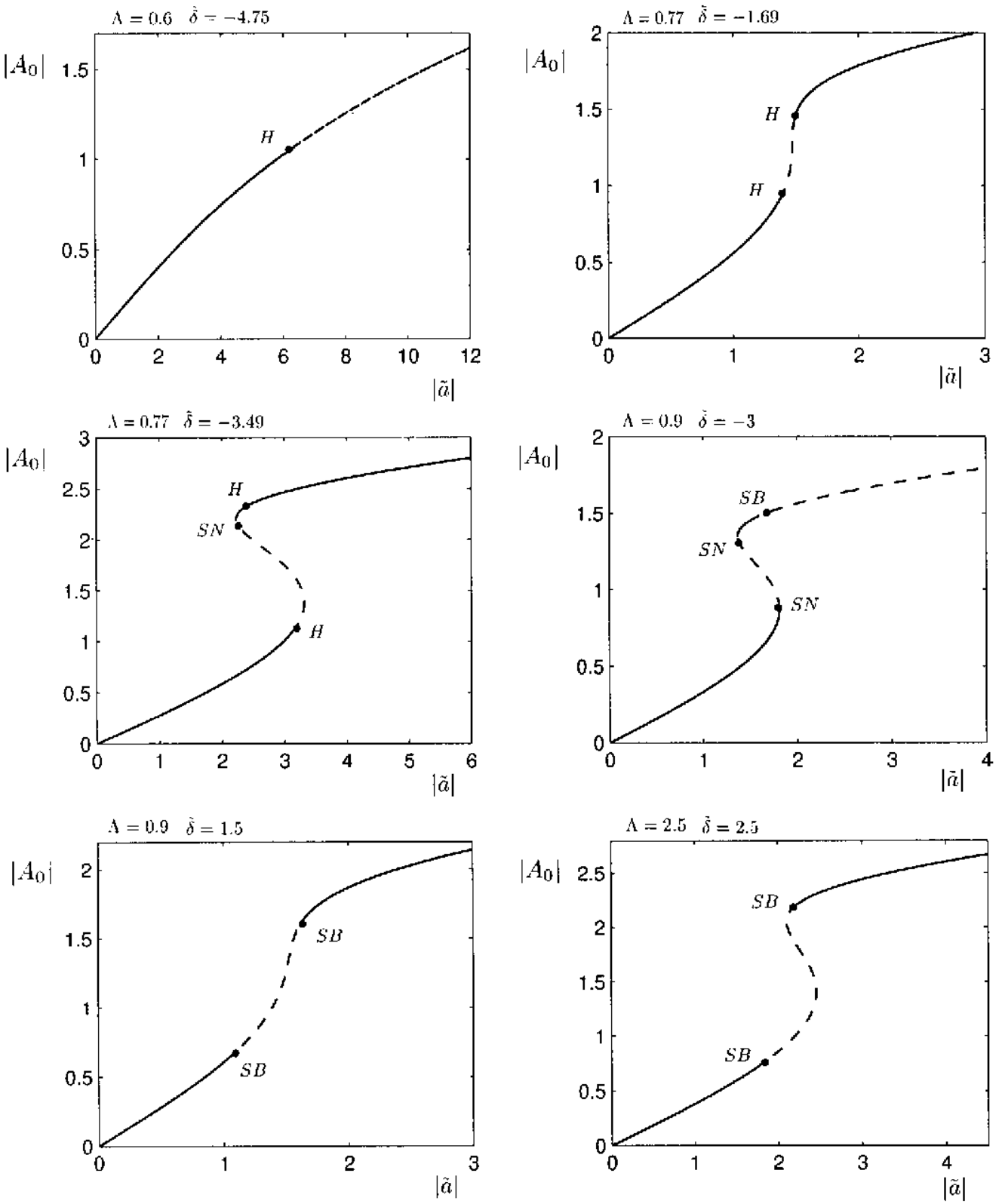

FIG. 6. The symmetric steady states of ACASF equations for $C=10^{-3}, n=1$ and the indieated values of $A$ and $\widetilde{\delta}$. Solid lines correspond to stable states and dashed to unstable.

After truncation, the complex amplitudes and the Galerkin coefficients are given by Eqs. (C9)-(C11), (C18) and (C19) [see also (C14)] in Appendix C, which are written for convenience as

$$
\begin{aligned}
A^{\prime}(\tau)= & (1 \mid \mathrm{i} \widetilde{\delta}) A \mid \mathrm{i}\left(\left.\alpha_{3}|A|^{2}\left|\alpha_{4}\right| B\right|^{2}\right) A \\
& -\mathrm{i} \gamma_{1} Z_{2} A+\mathrm{i} \widetilde{a}, \\
B^{\prime}(\tau)= & (1 \mid \mathrm{i} \widetilde{\delta}) B \mid \mathrm{i}\left(\left.\alpha_{3}|B|^{2}\left|\alpha_{4}\right| A\right|^{2}\right) B \\
& +\mathrm{i} \gamma_{1} Z_{2} B+\mathrm{i} \widetilde{a}, \\
Z_{1}^{\prime}(\tau)= & \lambda_{1} Z_{1}\left|\gamma_{2} Z_{2}^{2}\right| 2 \gamma_{3}\left|\hat{Z}_{3}\right|^{2} \mid \beta_{4}\left(|B|^{2} \quad|A|^{2}\right) Z_{2}, \\
Z_{2}^{\prime}(\tau)- & \lambda_{2} Z_{2}-\gamma_{2} Z_{1} Z_{2}+\beta_{2}\left(|B|^{2}-|A|^{2}\right), \\
\ell_{3}^{\prime}(\tau)- & \lambda_{3} \hat{Z}_{3}-\gamma_{3} Z_{1} \hat{Z}_{3},
\end{aligned}
$$

where the coefficients $\alpha_{3}, \alpha_{4}, \lambda_{1}, \lambda_{2}, \lambda_{3}, \gamma_{1}, \gamma_{2}, \gamma_{3}, \beta_{1}$, and $\beta_{2}$ are real. The eigenvalues of the three eigenmodes (i.e., $\lambda_{1}, \lambda_{2}$, and $\lambda_{3}$ ) are strictly negative, while the sign of the remaining cocflicients depend in principle on the parameters $\Lambda$ and $R_{s}$, and on the capillary mode being excited. These equations will be hereafter called truncated CASF $(T C A S F)$ equations, and are invariant under the symmetry

$$
A \leftrightarrow B, \quad Z_{2} \rightarrow-Z_{2}, \quad Z_{3} \rightarrow \overline{Z_{3}},
$$

which results from invariance of the original CASF equations under the azimuthal reflection (20).

Before describing the results of the numerical integrations on these equations, we note that the effect of the streaming flow on the capillary wave dynamics is small 
(a)

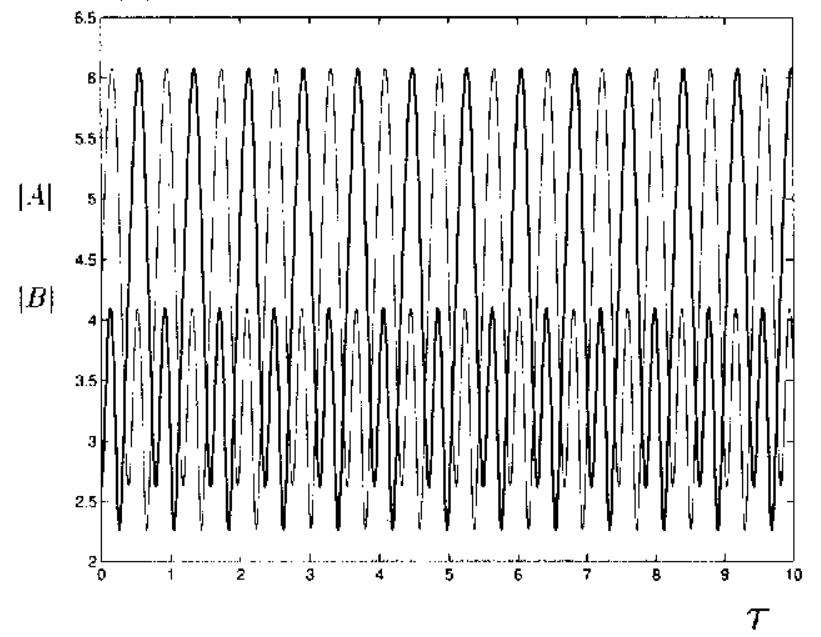

(b)

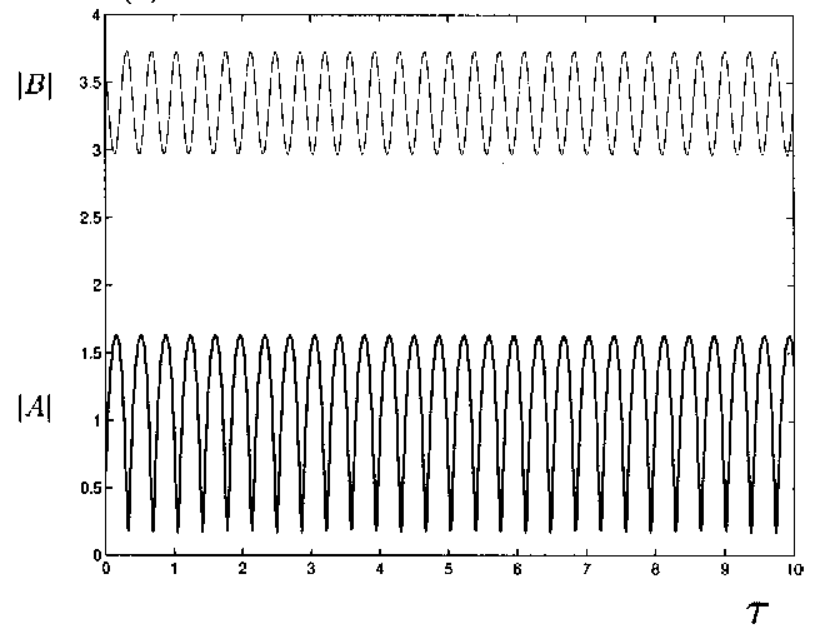

FIG. 7. $|A|$ (thick) and $|B|$ (thin) for stable periodic solutions of model (85)-(88), for $C=10^{3}$. (a) $\Lambda=0.7, \widetilde{\delta}=-6.85$ and $|\widetilde{a}|=20.59$ and (b) $\Lambda=0.6, \widetilde{\delta}$ 5 and $|\widetilde{a}| 20.45$.

whenever $\left|\gamma_{1} z_{2}\right| \ll\left(\left|\alpha_{3}\right|+\left|\alpha_{4}\right|\right)\left(|A|^{2}+|\beta|^{2}\right) / 2$, which requires, since $\left|Z_{2}\right| \cdots\left|\left(|B|^{2}-|A|^{2}\right) \beta_{2} / \lambda_{2}\right|$, that

$\left|\gamma_{1} \beta_{2}\left(|B|^{2}-|A|^{2}\right)\right| \ll\left|\lambda_{2}\right|\left(\left|\alpha_{3}\right|+\left|\alpha_{4}\right|\right)\left(|A|^{2}+|B|^{2}\right) / 2$.

This condition holds for reflection-symmetric solutions ( $A$ $-B$ ), as was to be expected, but for nonsy mmetric solutions can only hold if $2\left|\gamma_{1} \beta_{2}\right| /\left[\left|\lambda_{2}\right|\left(\left|\alpha_{3}\right|+\left|\alpha_{4}\right|\right)\right]$ is small. This parameter can be seen as a measure of the relative effect of the strcaming llow, and is illustrated in Fig. 8; the asymptotes result from the asymptotes of $\alpha_{3}$ and $\alpha_{4}$ (see Fig. 2). Thus the effect of the streaming flow can only be neglected for small $\Lambda$. In addition, the nonlinear terms can be neglected in Eqs. (93) $-(95)$ only if

$$
\frac{\left(\gamma_{2} \beta_{2}\right)^{2}}{\left|\lambda_{2}^{3} \lambda_{1}\right|}\left(|/ 3|^{2}-|A|^{2}\right)^{2} \ll 1
$$

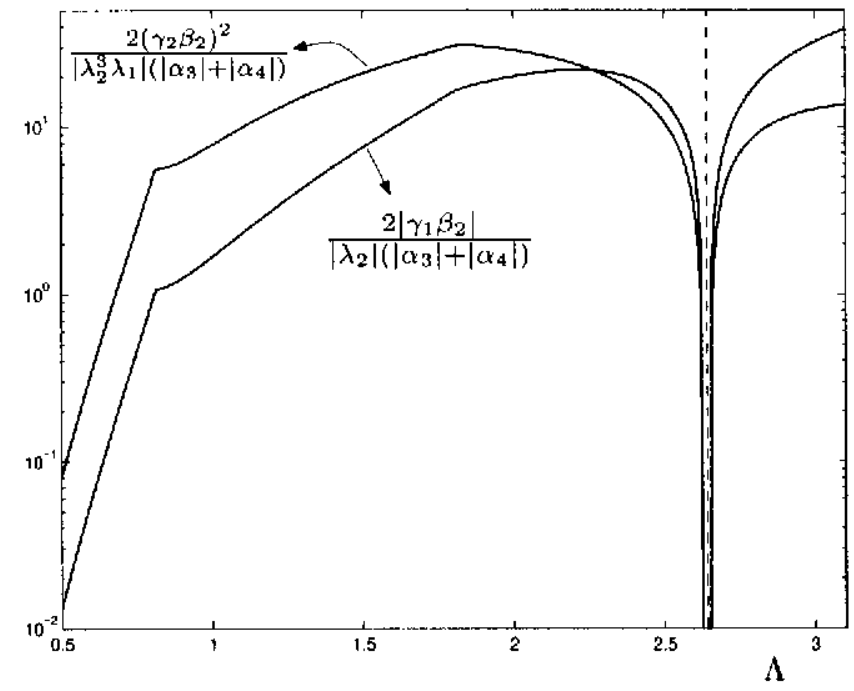

FIG. 8. Ratios $2\left|\gamma_{1} \beta_{2}\right|{ }^{\prime}\left[\left|\lambda_{2}\right|\left(\left|\alpha_{3}\right|+\left|\alpha_{1}\right|\right)\right]$ and $2 \mid\left(\gamma_{2} \beta_{2}\right)^{2}\left[\left|\lambda_{2}^{3} \lambda_{1}\right|\left(\mid \alpha_{3}\right.\right.$ $\left.\left.|+| \alpha_{4} \mid\right)\right\rceil$ is $\Lambda$, for the capillary mode $n=1$. as obtained taking into account that [sec Eq. (94)] $\left|Z_{1}\right|$ $\cdots\left|\gamma_{2} Z_{2}^{2} / \lambda_{1}\right|$ and $\left|Z_{2}\right| \cdots\left|\beta_{2}\left(|B|^{2}-|A|^{2}\right) / \lambda_{2}\right|$, and requiring that $\left|\gamma_{2} Z_{1}\right| \leqslant\left|\lambda_{2}\right|$. Condition (98) does hold for small wave stecpness and/or reflection-symmetric solutions. But otherwise it requires that the ratio $2\left(\gamma_{2} \beta_{2}\right)^{2} /\left|\lambda_{2}^{3} \lambda_{1}\left(\left|\alpha_{3}\right|+\left|\alpha_{4}\right|\right)\right|$ be small, which only happens (see Fig. 8) for small $\Lambda$; here we are assuming that $\widetilde{a} \sim 1$, which implies that $\left(|A|^{2}+|B|^{2}\right)$ $-2 /\left(\left|\alpha_{3}\right|+\left|\alpha_{4}\right|\right)$. All these mean that unfortunately conditions allowing to neglect nonlinear terms in Eqs. (93)-(95) but yiclding a significant role of the streaming llow are not easily met; namely, they require for $n-1$ that $\Lambda$ be small but not too small, say $0.6 \leqslant \Lambda \leqslant 0.7$. Therein Eq. (94) can be approximated as

$$
Z_{2}^{\prime}-\lambda_{2} Z_{2}+\beta_{2}\left(|B|^{2}-|A|^{2}\right)
$$

Equations (91), (92), (99) will be called truncated coupled amplitude-linear streaming flow (TCALSF) equations in the scquel.

\section{Analysis of the TCASF and TCALSF equations}

The primary bifurcation from the flat state in both systems, TCASF and TCALSF, yields a branch of SWs of the form $A-B-A_{0}, Z_{1}-Z_{2}-Z_{3}-0$, with $A_{0}$ satisfying (68). The linear stability of these yields results that are qualitatively similar to those obtained in Sec. III A 1. The growth rate $\lambda$ obcys again the characteristic cquations (76) and (77) for symmetric and antisymmetric perturbations, respectively, but with $\mathcal{H}$ replaced by

$$
\mathcal{H}-\gamma_{1} \beta_{2} /\left(-\lambda_{2}\right)
$$

We again obtain that the primary branch of SWs exhibits a Hopf bifurcation that is completely due to the streaming llow. This bifurcation is subcritical for all checked parameicr values. Therefore, any further analysis must rely on numerics.

The branch of periodic orbits produced in the Hopf bifurcation of the symmetric steady states is always unstable 
(a)

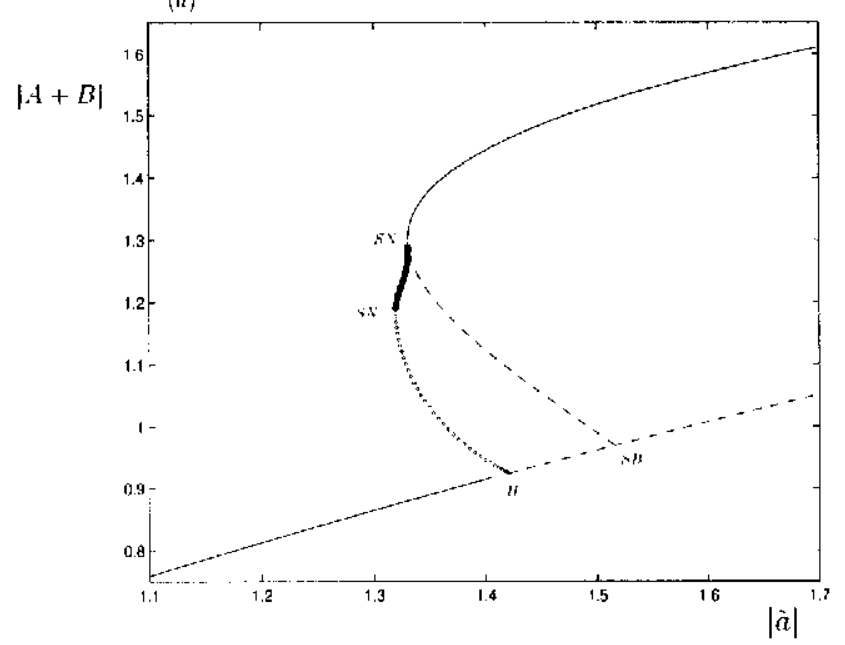

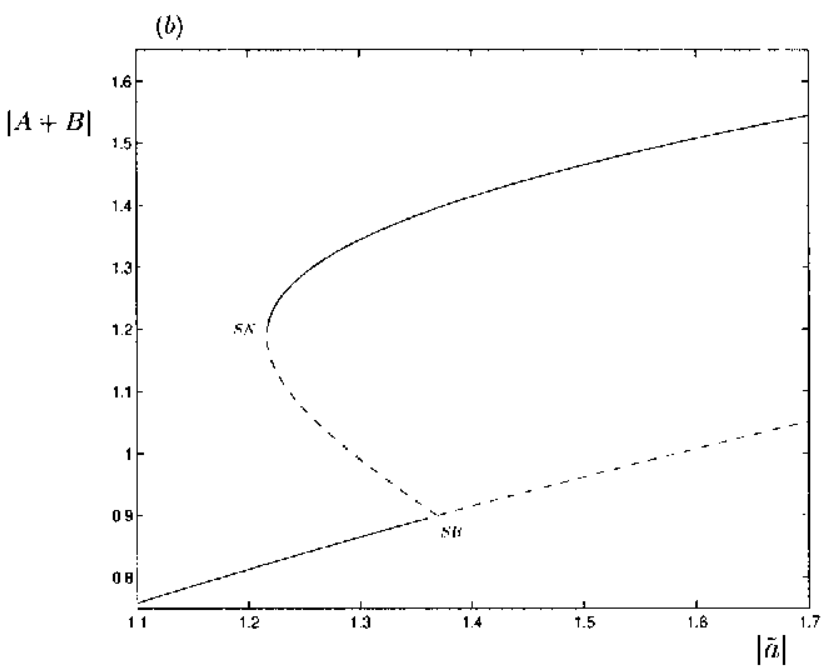

FIG. 9. Bifurcation diagrams for $\Lambda \quad 0.6$ and $\widetilde{\delta} \quad$ 2. (a) With streaming flow coupling and (b) without streaming flow. The lines comrespond to stable (solid) and unstable (dashed) steady solutions and the circles to stable (filled) and unstable (opened) periodic solutions.

near the onset. However, as one can see in Fig. 9(a), this unstable branch can undergo a saddle-node bifurcation, giving rise new stable periodic solutions, which eventually disappear in a homoclinic comnection (very near the saddlenode bifurcation of non-symmetric steady states). These periodic orbits are reflection-symmetric in phase space and correspond to blinking states (sce Fig. 10). In Fig. 9(b) we give the bifurcation diagram for Eqs. (91) and (92) with $\gamma_{1}$ $\equiv 0$ (i.e., in absence of streaming flow). Note that the dynamics of the counter-rolating capillary waves are indecd different when their interaction with the streaming flow is considered. However, the differences are evident only over a small interval of the forcing amplitude $|\widetilde{a}|$. In fact, according to our discussion above, for this value of the slenderness, $\Lambda$ -0.6 , the TCALSF cquations should apply, but the efrect of the streaming flow should not be quite strong. As shown the bifurcation diagrams of Fig. 11, the effect of the streaming flow is still relatively weak for $\Lambda-0.7$. Since these diagrams involve chaotic dynamics we plot in Fig. $12(|B|-|A|)$ vs $|\widetilde{a}|$ for those points of the orbit where $\left.|A|^{2}|| B\right|^{2}$ gets a local maximum, which are given by the outward intersections of attractors with the Poincare hypersurface: $2\left(|A|^{2}+|B|^{2}\right)$ $-\mathrm{i} \widetilde{a}(\bar{A}+\bar{B})+$ c.c. (a cylinder in the phase space); this hypersurface follows from the exact relation:

$$
\begin{aligned}
& \frac{d}{d \tau}\left(\left.|A|^{2}|| B\right|^{2}\right)= 2\left(\left.|A|^{2}|| B\right|^{2}\right) \\
&+[\mathrm{i} \widetilde{a}(\bar{A}+\bar{B})+\text { c.C. }],
\end{aligned}
$$

which is obtained readily from (91) and (92). Both systems. with and without streaming flow, exhibit qualitatively similar (slable) dynamics. As secn in Fig. 11 the primary branch of SWs loses stability again either at a subcritical Hopf bifurcation (wilh strcaming llow) or at a subcritical symmetry breaking bifurcation (without streaming flow), where the system jumps to a branch of nonsy mmetric steady states; this occurs in both systems at roughly the same value of $\tilde{a}$ ( $\tilde{a}$ $\approx 4.19)$. Note that this transition involves significant hyster- esis. The nonsymmetric steady solutions then undergo a subcritical Hopf bifurcation and a branch of reflectionsymmetric periodic solutions is observed. Here the quantitative effect of the streaming flow is significant, and again there is hysteresis in both cases. As $\widetilde{a}$ increases, the periodic branch becomes unstable in a subcritical, period-1, symmetry-brcaking bifurcation and chaotic states can be observed through a type I intermittency. The chaotic attractor is symmetric on average (Fig. 12), and exhibits narrow periodic windows not appreciated in this not-detailed-cnough plot. The chaotic attractor loses stability at a higher value of $\widetilde{a}$ and the symmetric steady states are recovered.

For larger values of $\widetilde{a}$ and/or $\Lambda$ nonlinear terms in Eqs. (93)-(95) cannot be ignored according to our discussion above $[\mathrm{cl} .(97)-(98)]$, and the TLCASF equations do not apply. As an illustration, we plot some bifurcation diagrams for $\Lambda=0.7$ and $\Lambda=0.9$ in Fig. 13 corresponding to three system of equations: (i) TCASF cquations (91)-(95), (ii) the

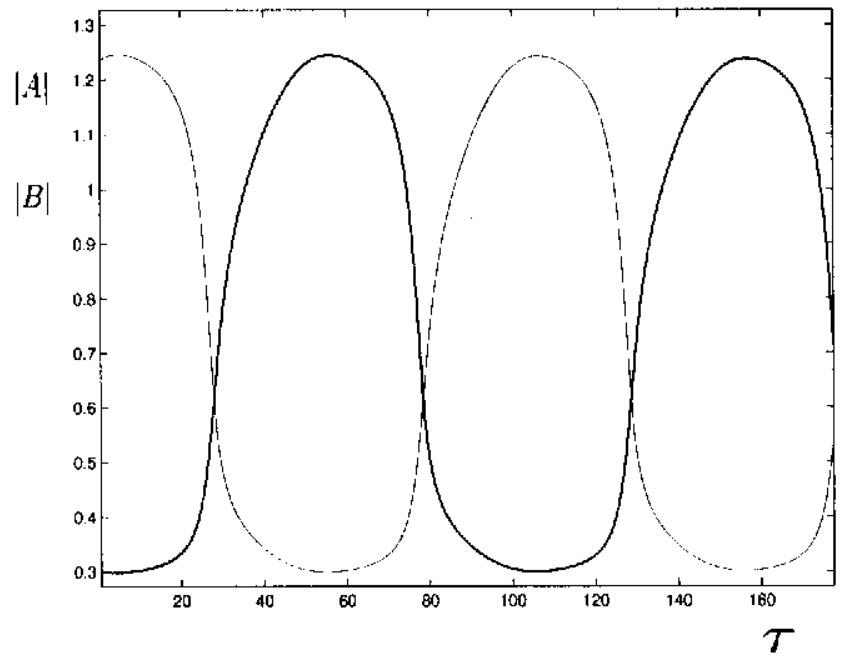

FIG. 10. $|A|$ (thick) and $|B|$ (thin) for at stable periodic solution of the TCALSF equations (91) and (92), and (99) for $\Lambda=0.6$ and $\widetilde{\delta}=-2$. 
(a)

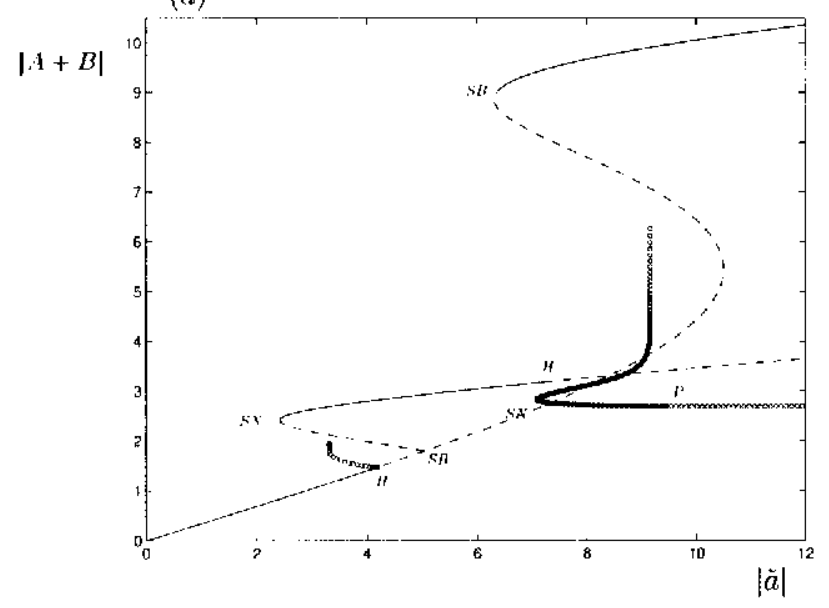

(b)

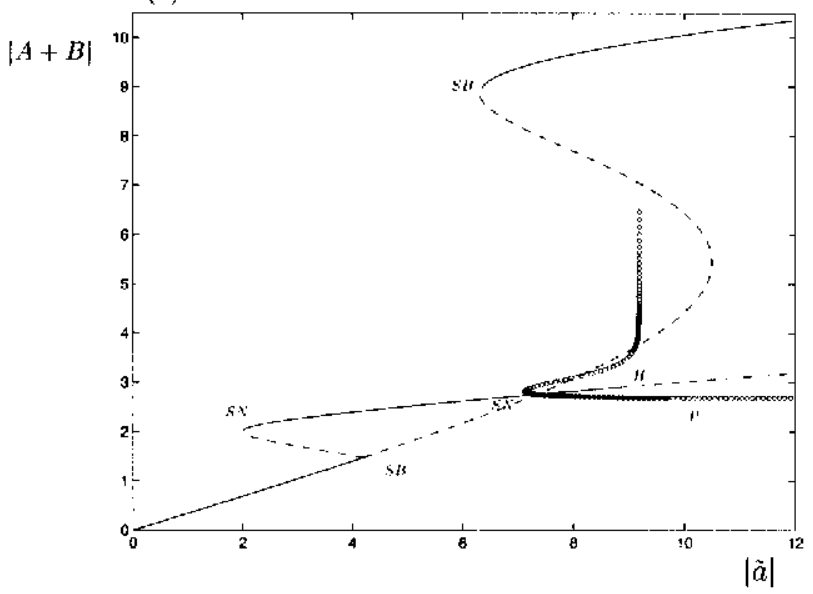

ГIG. 11. As in Fig. 9 but for $\Lambda=0.7$ and $\widetilde{\delta}=-4$.

TCALSF equations (91)-(92) and (99) and (iii) the amplitude equations $(91)-(92)$ with $\gamma_{1}-0$. Note that for $\Lambda-0.9$ condition (98) is not satisfied and nonlinear terms must be included even for $\tilde{a}-1$. These terms tend to stabilize the primary branches and eliminate the region of complex dynamics displayed by Eqs. (91), (92) and (99).

\section{PARAMETRIC FORCING}

In the preceding sections we have only considered nonaxisymmetric eigenmodes with aximulhal wave number $m=1$. This has been so because the nature of the external vibrations, see Eq. (5). And the same would be true for any other external vibration at a frequency close to a natural frequency. Parametric forcing, ${ }^{31,32}$ due to external vibrations at a frequency close to twice a natural frequency, allows to excite any nonaxisymmetric mode in a natural way. If, in addition, the external vibrations do not break invariance under rotation (e.g., axial vibration of one disk), then the streaming flow equations (61)-(64) stand after replacing $\mathrm{e}^{2 \mathrm{i} \theta}$ by $\mathrm{e}^{2 \mathrm{im} \theta}$, where $m$ is the azimulhal wave number. The amplitude cquations (59) and (60) instead must be replaced by

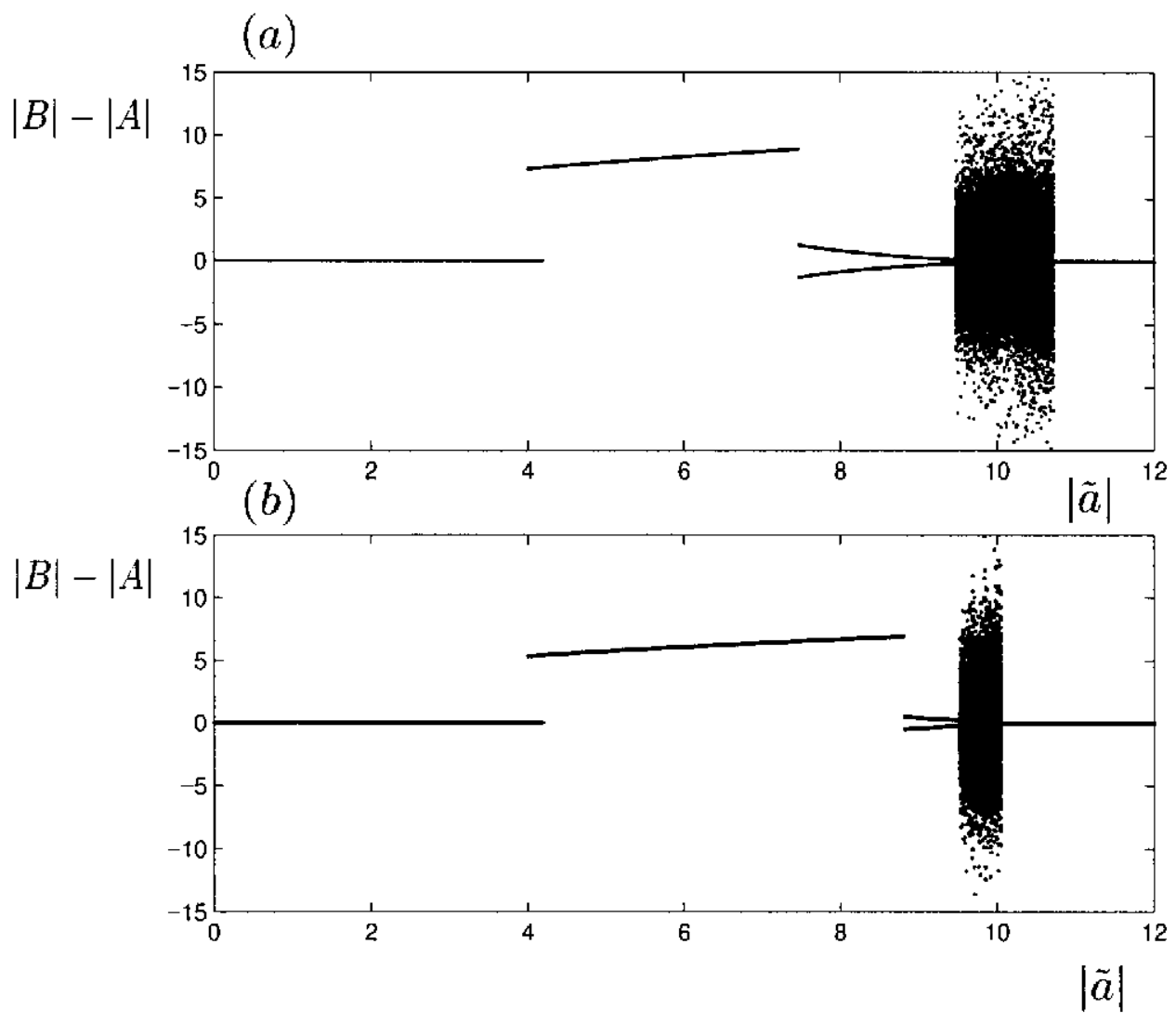

FIG. 12. Bifurcation diagrams of the TCALSF equations resulting from the Poincare map for $\Lambda=0.7$ and $\tilde{\delta}=-4$. (a) With strcaming flow, and (b) without streaming flow. 

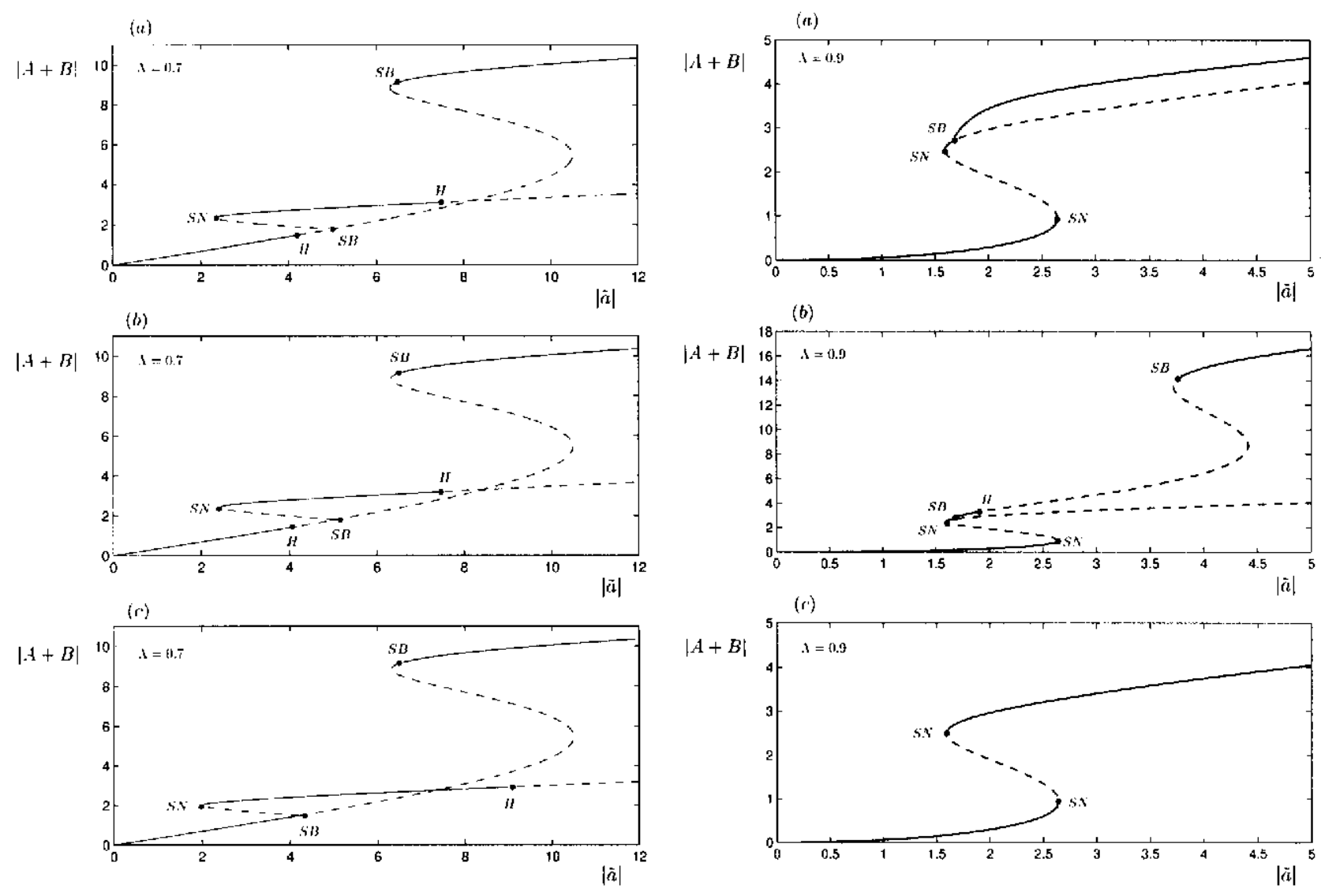

FIG. 13. Bifurcation diagrams for $\Lambda \quad 0.7$ and $\widetilde{\delta} \quad 4$ (left) and for $\Lambda \quad 0.9$ and $\widetilde{\delta} \quad 4$ (right). (a) Original TC $\Lambda$ SF equations (91) (95), (b) TC $A$ LSF equations (91)-(92) and (99), and (c) amplitude equations (91)-(92) with $\gamma_{1}=0$.

$$
\begin{aligned}
A^{\prime}- & -(1+\mathrm{i} \widetilde{\delta}) A+\mathrm{i}\left(\alpha_{3}|A|^{2}+\alpha_{4}|B|^{2}\right) A \\
& -\mathrm{i} \alpha_{5} \int_{\Sigma_{0}} \boldsymbol{g} \cdot \boldsymbol{v} d \boldsymbol{x} A+\mathrm{i} \widetilde{a} \beta, \\
B^{\prime}= & (1 \mid \mathrm{i} \widetilde{\delta}) B \mid \mathrm{i}\left(\left.\alpha_{3}|B|^{2}\left|\alpha_{4}\right| A\right|^{2}\right) B \\
& \left|\mathrm{i} \alpha_{5} \int_{\Sigma_{0}} \boldsymbol{g} \cdot \boldsymbol{v} d \boldsymbol{x} B\right| \mathrm{i} \widetilde{a} \bar{A},
\end{aligned}
$$

where $\widetilde{a}$ is a re-scaled forcing acceleration. These amplitude equations are similar to the ones that apply to Faraday waves, ${ }^{32}$ except for the streaming flow coupling that is always (inconsistently, see Ref. 22) ignored in the analysis of these waves. These equations are further simplified using the new variables

$$
\widetilde{A}-A \mathrm{e}^{\mathrm{i} m \psi}, \quad \widetilde{B}-B \mathrm{e}^{-\mathrm{i} m \psi},
$$

where the spatial phase $\psi$ is given by

$$
\frac{d \psi}{d \tau}-\frac{\alpha_{5}}{m} \int_{\Sigma_{0}} g \cdot v d \boldsymbol{x}
$$

which reduce (101) and (102) to

$$
\begin{aligned}
& \widetilde{A}^{\prime}--(1+\mathrm{i} \widetilde{\delta}) \widetilde{A}+\mathrm{i}\left(\alpha_{3}|\widetilde{A}|^{2}+\alpha_{4}|\widetilde{B}|^{2}\right) \widetilde{A}+\mathrm{i} \widetilde{a} \tilde{B}, \\
& \widetilde{B}^{\prime}--(1+\mathrm{i} \widetilde{\delta}) \widetilde{B}+\mathrm{i}\left(\alpha_{3}|\widetilde{B}|^{2}+\alpha_{4}|\widetilde{A}|^{2}\right) \widetilde{B}+\mathrm{i} \widetilde{a} \tilde{A} .
\end{aligned}
$$

All solutions of these two equations are well known to converge to the set of steady states, which are such that $|\widetilde{A}|$ $-|\widetilde{\beta}|$. Ignoring the transient when this convergence takes place, we can assume that

$$
\widetilde{A}-\widetilde{B}-R_{0} \mathrm{e}^{\mathrm{im} \phi_{0}}-\text { constant, }
$$

where we have used invariance of (105) and (106) under rotations $\widetilde{A} \rightarrow \widetilde{A} \mathrm{e}^{i c}, \widetilde{B} \rightarrow \widetilde{B} \mathrm{e}^{i c}$ to eliminate the difference between the phases of $\widetilde{A}$ and $\widetilde{B}$. And we only need to replace (103) and (107) into (61)-(64) to obtain

$$
\begin{aligned}
& \nabla \cdot \boldsymbol{v}=0, \\
& \partial \boldsymbol{v} / \partial \tau-\boldsymbol{v} \times(\nabla \times \boldsymbol{v})--\nabla \widetilde{q}+R_{s}^{-1} \Delta \boldsymbol{v} \quad \text { in } \Sigma_{0}, \\
& \boldsymbol{v}-2 R_{0}^{2}\left[\varphi_{1} \boldsymbol{e}_{r}+\varphi_{2} \cos [2 m(\theta-\psi /)] \boldsymbol{e}_{r}\right. \\
& \left.\quad+\varphi_{3} \sin [2 m(\theta-\psi)] \boldsymbol{e}_{0}\right] \text { at } z^{-} \pm \Lambda, \\
& \boldsymbol{v} \cdot \boldsymbol{e}_{r}-0, \quad(\partial \boldsymbol{v} / \partial r-\boldsymbol{v}) \cdot \boldsymbol{e}_{0}-0 \\
& (\partial \boldsymbol{v} / \partial r) \cdot \boldsymbol{e}_{z}-0 \quad \text { at } r-1 .
\end{aligned}
$$

Note that these solutions are standing waves in a rotated reference frame, such that the azimuthal coordinate is $\theta=\psi$ for, using (23), (103), and (107), the radial free surface deflection is given by

$$
f-1+4 \varepsilon^{2} R_{0} \cos (\omega t) \cos [2 m(\theta-\psi)]+\cdots .
$$


Thus these waves are completely determined up to the phase 1/, whose evolution is coupled to the streaming flow, through Eqs. (104), (108)-(111). The simplest nontrivial solutions to these equations are steadily rotating. quasi-standing waves of the form

$$
\psi-c \tau, \quad \boldsymbol{v}-\boldsymbol{v}(\boldsymbol{r}, \theta-c \tau, z)+c r \tau \boldsymbol{e}_{\theta} .
$$

We say that the wave is quasi-standing because the nodal lines move but only on the slow time scale $\tau$, for such waves the phase of $A\left(\boldsymbol{V}_{0}^{1}, Q_{0}, F_{0}\right) \mathrm{e}^{\mathrm{i} m \theta} \mid B\left(\boldsymbol{V}_{0}, Q_{0}, F_{0}\right) \mathrm{e}^{-\mathrm{i} m \theta}$ is independent of the position (but will depend on $\tau)$. The existence and stability of the rotating waves (104), (108)-(111) is beyond the scope of this paper. Some recent results on the two-dimensional version of this problem ${ }^{33}$ suggest that both stable rotating waves and more complex attractors are to be cxpecled.

The reduction above to rotating quasi-standing waves was possible because of the invariance under rotation of the problem. If this is broken by, c.g., making the disks slightly noncircular but still reflection symmetric, then the two counterpropagating modes split into two modes with different cigenfrequencies. As a consequence, the amplitude equations (105) and (106) must be replaced by

$$
\begin{aligned}
& A^{\prime}--\left(1+\mathrm{i} \widetilde{\delta}_{1}\right) A+\mathrm{i} \widetilde{\delta}_{2} \widetilde{B}+\mathrm{i}\left(\alpha_{3}|A|^{2}+\alpha_{4}|B|^{2}\right) A \\
& -\quad \mathrm{i} \alpha_{5} \int_{\Sigma_{0}} \boldsymbol{g} \cdot \boldsymbol{v} d \boldsymbol{x} A+\mathrm{i} \widetilde{a} \bar{B}, \\
& B^{\prime}--\left(1+\mathrm{i} \widetilde{\delta}_{1}\right) B+\mathrm{i} \widetilde{\delta}_{2} A+\mathrm{i}\left(\alpha_{3}|B|^{2}+\alpha_{4}|A|^{2}\right) B \\
& \quad-\mathrm{i} \alpha_{5} \int_{\Sigma_{0}} \boldsymbol{g} \cdot \boldsymbol{v} d \boldsymbol{x} B+\mathrm{i} \tilde{a} \bar{A},
\end{aligned}
$$

where $\widetilde{\delta}_{1}$ and $\widetilde{\delta}_{2}$ are the scaled detunings associated with the two capillary modes, which are now different from each other. In fact, $\widetilde{\delta}_{1}-\widetilde{\delta}_{2}$ can be seen as a scaled [according to (58)] measure of departure from the rotationally symmetric case. Some experimental results on the dramatic effect of breaking the square symmetry in the Faraday experiment ${ }^{34,35}$ suggests that the effect of breaking the rotational symmetry in the liquid bridge may have a profound effect on dy namics for $\left|\widetilde{\delta}_{1}-\widetilde{\delta}_{2}\right| \rightarrow 1$, which means that the disks are only slightly noncircular. But no experiments are available to confirm this conjecture, and the analysis of the associated equations, (61)-(64), (114) and (115) is well outside the scope of this paper.

\section{CONCLUSIONS}

We have considered the weakly nonlinear response of nearly inviscid capillary bridges to nonaxisymmetric direct periodic excitation with a frequency close to a natural frequency. We have seen that the streaming flow produced by the capillary waves leads to an effect on the capillary waves themselves that is of the same order as the cubic terms that are always retained in weakly nonlinear theories. Thus the streaming flow cannot be ignored. We have derived some general amplitude-streaming flow equations that account for the coupled evolution of the counter-rotating waves amplitudes and the streaming flow velocity ficld. These equations still include a Navier-Stokes-type equation, but are much simpler than the original formulation because: (a) The fast oscillation has been filtered out, (b) the oscillatory boundary layers atlached to the disks and the free surface have been solved, and their effect included as appropriate boundary conditions, which (c) are imposed at the fixed unperturbed free surface, instead of at the moving free surface. We have presented several simplificd equations that are more amenable to analytical and numerical treatments. The analysis of these has led to several predictions that could be used for comparison with experiments. In particular, we have analyzed the stability of the simplest monochromatic standing waves, which exhibit both nonoscillatory and oscillatory instabilities, the latter being entirely due to the effect of the streaming flow. Nonoscillatory instabilities led to (a) nonreflection symmetric steady states of the amplitude equations that correspond to a kind of rotating states in which one of the counter-rotating waves dominates. The Hopf bifurcation instcad gave rise to (b) rellection symmelric periodic orbits that correspond to blinking states in which each wave dominates in a part of the period. In addition we have encountered (c) nonsymmetric periodic orbits that correspond again to a kind of rotating states, and (d) chaotic attractors. In general, any nonrellection symmetric solution of the CASF equations is affected by the streaming flow. The quantitative effect of the streaming flow in the amplitude equations has been estimated to obtain that it is generally significant, cxcept for small slenderness (cf. Fig. 8). Likewise the effect of convective terms in the streaming flow momentum equation has also been evaluated to conclude that these terms can be neglected only for moderately small values of the slenderness, $0.6 \% \Lambda \leqslant 0.7$ (cl. Fig. 8).

For simplicity, we have considered a forcing device that always preserves reflection symmetry with respect to a plane containing the symmetry axis. But this symmetry can be broken from the outset by, e.g., rotating the symmetry axis around a horizontal circle, with constant angular velocity. In this case, one of the counter-rotating waves dominates and some transitions observed above disappear. This paper has been mostly devoted to direct forcing, at a frequency close to a natural frequency. However, that device was effective only to excite that mode with azimuthal wave number $m=1$ (in addition, of course to the axisy mmetric modes corresponding to $m-0$ ), already considered in Refs. 14 and 15 . In order to excite (effectively) eigenmodes with wave number $m \neq 0,1$, we have also briclly considered oscillations of the capillary bridge excited by parametric forcing. at a frequency close to twice a natural frequency. We have shown that because of invariance under rotation, all solutions of the relevant CASF equations evolve to rotating quasi-standing waves, which are determined up to a phase shift that couples with the streaming flow. We have obtained the relevant equations for this interaction, and explained the nature of the expected solulions.

The results above show that forced nonaxisymmetric os- 
cillations in capillary bridges between circular disks exhibit a variety of complex large time behaviors, beyond the simplest monochromalic slanding waves, whose description requires to consider the effect of the streaming flow produced by the waves on the waves themselves. Because of the lack of experiments on nonlinear, nonaxisymmetric capillary waves we have been unable to make any comparison. Experimental visualizations and measurements of the associated mean llow are also much needed.

\section{ACKNOWLEDGMENTS}

This research was supported by the Spanish Dirección General de Investigación, under Grant No. BFM2001-2363 and by the NASA Microgravily Science Program under Grant No. NAG3-2152.

\section{APPENDIX A: SOME USEFUL EXPRESSIONS}

The linear eigenvalue problem (24)-(26) has nontrivial solutions if and only if one of the following dispersion relations hold, which correspond to odd and even modes, respectively

$$
\begin{aligned}
& \Lambda^{2}+\sum_{n \text { odd }} a_{n}-0, \\
& 1 / \Omega^{2}\left|\Lambda^{2} / 3\right| \sum_{n \text { even }} a_{n}=0,
\end{aligned}
$$

where

$$
a_{n}--2 \Omega^{2} I_{1}\left(l_{n}\right) /\left[\Omega^{2} l_{n}^{2} I_{1}\left(l_{n}\right)+l_{n}^{5} I_{1}^{\prime}\left(l_{n}\right)\right] .
$$

with $l_{n}-n \pi / 2 \Lambda$. Here, $I_{1}$ is the modified Bessel function of first kind and order one. If (A1) holds then $Q_{0}$ and $F_{0}$ are odd functions of $z$ and (up to a constant complex factor) are given by

$$
\begin{aligned}
& Q_{0}--\sum_{n o d a l} l_{n}^{2} a_{n}\left[l_{1}\left(l_{n} r\right) / I_{1}\left(l_{n}\right)\right] \cos \left[l_{n}(z+\Lambda)\right], \\
& F_{0}-\Lambda z-\sum_{n \text { odd }} a_{n} \cos \left[l_{n}(z+\Lambda)\right] .
\end{aligned}
$$

The velocity field $\boldsymbol{v}_{0}$ is readily calculated from the second cquation (24). Similarly, when (A1) holds $Q_{0}$ and $F_{0}$ are even functions of $z$ and are given by

$$
\begin{aligned}
& Q_{0}-r-\sum_{n \text { even }} l_{n}^{2} a_{n}\left[I_{1}\left(l_{n} r\right) / I_{1}\left(l_{n}\right)\right] \cos \left[l_{n}(z+\Lambda)\right], \\
& F_{0}-\Lambda^{2} / 6-1 / \Omega^{2}-z^{2} / 2-\sum_{n \text { even }} a_{n} \cos \left[l_{n}(z+\Lambda)\right] .
\end{aligned}
$$

The formula from vector-analysis invoked in Sec. II B to obtain (54) is given in the following:

Lemma. Let $\boldsymbol{u}$ be a complex vector field and let $\boldsymbol{w}$ be a real vector field defined in the three-dimensional, real tiuclidean space. If $\boldsymbol{\nabla} \cdot \boldsymbol{u}-0$ and $\boldsymbol{\nabla} \times \boldsymbol{u}-\mathbf{0}$ then we have

$\mathrm{i} \boldsymbol{u} \times \nabla \times[\bar{u} \times(\nabla \times \boldsymbol{w})]$ । c.c.

$$
-\mathrm{i}[\boldsymbol{\nabla} \times(\boldsymbol{u} \times \bar{u})] \times(\boldsymbol{\nabla} \times \boldsymbol{w})+\mathrm{i} \boldsymbol{\nabla}[(\boldsymbol{\nabla} \times \boldsymbol{w}) \cdot(\boldsymbol{u} \times \bar{u})],
$$

where (as above) we are using the complex inner product defined in (35).

Proof. When using Cartesian coordinates, the / th coordinate of $v \times(\nabla \times w)$ is given by

$$
[\boldsymbol{v} \times(\boldsymbol{\nabla} \times w)]_{l}-v_{j}\left(\partial_{i} w_{j}-\partial_{j} w_{l}\right)
$$

for any pair of (real or complex) vector fields $v$ and $w$; here we are using the Finstein summation comection about the summation over repeated indexes and $\partial_{l}$ stands for the partial derivative with respect to the Cartesian coordinate $x_{l}$. Also, since the vector field $\boldsymbol{u}$ is solenoidal we have $\nabla \times(\bar{u} \times \boldsymbol{u})$ $=(\bar{u} \cdot \nabla) \boldsymbol{u}(\boldsymbol{u} \cdot \nabla) \bar{u}$ and, since $\boldsymbol{u}$ is also irrotational, the $l$ th coordinate of $\boldsymbol{\nabla} \times(\overline{\boldsymbol{u}} \times \boldsymbol{u})$ is given by

$$
[\nabla \times(\overline{\boldsymbol{u}} \times \boldsymbol{u})]_{l}=\overline{u_{j}} \partial_{j} u_{l} \quad u_{j} \partial_{j} \overline{u_{l}}=\overline{u_{j}} \partial_{l} u_{j} \quad u_{j} \partial_{l} \overline{u_{j}} .
$$

Finally, when taking into account that $(\nabla \times w) \cdot(u \times \bar{u})$ $=\boldsymbol{u} \cdot[\boldsymbol{u} \times(\nabla \times w)]$, and using (A8) we obtain

$$
(\nabla \times \boldsymbol{w}) \cdot(\boldsymbol{u} \times \overline{\boldsymbol{u}})-\bar{u}_{k} u_{j}\left(\partial_{j} w_{k}-\partial_{k} w_{j}\right) .
$$

Then we only need to use (A8)-(A10) to obtain (after some algebra) that the $p$ th Cartesian component of both sides of (A7) coincides. Thus the proof is complete.

\section{APPENDIX B: THE COEFFICIENTS $O\left(\varepsilon^{2}\right)$ PROBLEM IN THE EXPANSIONS (22) and (23)}

This problem is given by

$$
\begin{aligned}
& \nabla \cdot \boldsymbol{v}_{2}-0, \quad \boldsymbol{v}_{2 t}+\nabla q_{2}-0, \\
& \text { in } \quad 0 \leqslant<1, \quad|z|<\Lambda, \\
& \boldsymbol{v}_{2} \cdot \boldsymbol{e}_{z}-0, \quad f_{2}-0 \quad \text { at } z-\mid \Lambda \\
& \boldsymbol{v}_{2} \cdot \boldsymbol{e}_{r}-f_{2 t}- {\left[\mathrm{e}^{\mathrm{i} \Omega t}\left(A \boldsymbol{v}_{0}^{+}+B \boldsymbol{v}_{0}^{-}\right)+\mathrm{c} . \mathrm{c} .\right] } \\
& \times\left[\mathrm{e}^{\mathrm{i} \Omega t}\left(\left(A f_{0 \ell} \mid B f_{00}^{-}\right) \boldsymbol{e}_{\theta} \mid\left(A f_{0 \mathrm{i} z}^{+} \mid B f_{0 z}^{-}\right) \boldsymbol{e}_{z}\right)\right. \\
&+\mathrm{c.c} .]-\left[\mathrm{c}^{\mathrm{i} \Omega t}\left(A f_{0}^{-}+B f_{0}\right)+\mathrm{c.c} .\right] \\
& \times\left[\mathrm{e}^{\mathrm{i} \Omega t}\left(A \boldsymbol{v}_{0 r}^{+}+B \boldsymbol{v}_{0 r}^{-}\right) \cdot \boldsymbol{e}_{r}+\text { c.c. }\right] \quad \text { at } r-1,
\end{aligned}
$$

$$
\begin{aligned}
& q_{2}\left|f_{2}\right| f_{2 \ell \theta} \mid f_{2 z z} \\
& =\left[\mathrm{e}^{\mathrm{i} \Omega t}\left(A \boldsymbol{v}_{0} \mid B \boldsymbol{v}_{0}\right) \mid \text { c.c. }\right]^{2} / 2 \\
& +\left[\mathrm{c}^{\mathrm{i} \Omega t}\left(A \int_{0}+B f_{0}\right)+\mathrm{c.c} .\right]^{2} \\
& +\left[\mathrm{e}^{\mathrm{i} \Omega t}\left(A f_{0 \theta}^{-}+B f_{0 \theta}^{-}\right)+\text {c.c. }\right]^{2} / 2 \\
& {\left[\mathrm{e}^{\mathrm{i} \Omega t}\left(A f_{0 z} \mid B f_{0 \Sigma}^{-}\right) \mid \text {c.c. }\right]^{2} / 2} \\
& \text { | } 2\left[\mathrm{e}^{\mathrm{i} \Omega t}\left(A f_{0}^{\prime} \mid B f_{0}\right) \mid \text { c.c. }\right]\left[\mathrm{e}^{\mathrm{i} \Omega t}\left(A f_{O \theta \theta}^{\prime} \mid B f_{O \theta \theta}\right) \mid \text { c.c. }\right] \\
& -\left[\mathrm{c}^{\mathrm{i} \Omega t}\left(A f_{0 j}^{-}+B f_{0}^{-}\right)+\mathrm{c.c} .\right] \\
& \times\left[\mathrm{e}^{\mathrm{i} \Omega l}\left(A q_{\hat{\omega} r}^{+}+B q_{i r r}^{-}\right)+\mathrm{c.c} .\right] \quad \text { at } r-1, \\
& \int_{0}^{2 \pi / \Omega} \int_{-\Lambda}^{\Lambda} f_{2} d \theta d z=\int_{0}^{2 \pi / \Omega} \int_{-\Lambda}^{\Lambda}\left[\mathrm{e}^{\mathrm{i} \Omega}\left(A f_{0}^{+} \mid B f_{0}^{-}\right)\right. \\
& + \text {c.c.] } d \theta d z \text {, } \\
& \int_{0}^{2 \pi / \Omega} \int_{-\Lambda}^{\Lambda} \int_{0}^{2 \pi} \int_{0}^{1} \boldsymbol{v}_{2} \cdot \boldsymbol{v}_{0}^{ \pm} \mathrm{c}^{-\mathrm{i} \Omega t} r d r d \theta d z d t-0,
\end{aligned}
$$


where $\boldsymbol{v}_{0}^{ \pm}, q_{0}^{ \pm}$, and $f_{0}^{ \pm}$are as given by (27) and the parlial derivatives are denoted by subscripts. This problem is obtained upon substitution of (22) and (23) and (31) into (2), (3), (6)-(7), and (9), and setting to zero the coefficients of $\varepsilon^{2}$. The last determinacy condition eliminates any component of the solution of the form $\mathrm{e}^{ \pm i \Omega t}\left(v_{0}^{\frac{1}{0}}, q_{0}^{\frac{1}{0}}, f_{0}^{\frac{1}{1}}\right)$, which would appear as solution of the homogencous problem. The solution of (B1)-(B6) can be written as

$$
\begin{aligned}
& \boldsymbol{v}_{2}=\mathrm{e}^{2 i \Omega l}\left(A^{2} \boldsymbol{V}_{22}^{+} \mathrm{e}^{2 i \theta} \mid B^{2} \boldsymbol{V}_{22}^{-} \mathrm{e}^{2 \mathrm{i} \theta}\right) \mid \mathrm{e}^{2 i \Omega t} A B \boldsymbol{V}_{20} \text { | c.c., } \\
& q_{2}=\mathrm{e}^{2 i \Omega t} Q_{22}\left(A^{2} \mathrm{e}^{2 \mathrm{i} \theta} \mid B^{2} \mathrm{e}^{-2 \mathrm{i} \theta}\right) \mid \mathrm{e}^{2 \mathrm{i} \Omega t} A B Q_{2 i} \\
& +\mathrm{e}^{2 i \theta} A \bar{B} Q_{02}+\text { c.c. }+\left(|A|^{2}+|B|^{2}\right) Q_{00}, \\
& f_{2}-\mathrm{c}^{2 \mathrm{i} \Omega t} F_{22}\left(A^{2} \mathrm{c}^{2 \mathrm{i} \theta}+B^{2} \mathrm{c}^{-2 \mathrm{i} \theta}\right)+\mathrm{c}^{2 \mathrm{i} \Omega t} A B F_{20} \\
& \text { | } \mathrm{e}^{2 \mathrm{i} \theta} A \overline{B F_{02}} \text { | c.c. } \mid\left(\left.|A|^{2}|| B\right|^{2}\right) F_{001} \text {, }
\end{aligned}
$$

with

$$
\begin{aligned}
& \boldsymbol{V}_{22}^{\dot{\prime}}=U_{22} \boldsymbol{e}_{r} \pm V_{22} \boldsymbol{e}_{\theta} \mid W_{22} \boldsymbol{e}_{z}, \\
& \boldsymbol{V}_{20}=U_{20} \boldsymbol{e}_{r} \mid W_{20} \boldsymbol{e}_{z} .
\end{aligned}
$$

Here, $\left(\boldsymbol{V}_{22}^{\perp}, Q_{22}, F_{22}\right), \quad\left(\boldsymbol{V}_{20}, Q_{20}, F_{20}\right), \quad\left(Q_{02}, F_{02}\right)$, and $\left(Q_{00}, F_{i 01}\right)$ are independent of $t$, and are numerically calculated (by, e.g., a spectral method) from some $2 \mathrm{D}$ nonsingular linear problems obtained upon substitution of (B7)-(B9) into (B1)-(B6). Herc we just point out that

$$
\boldsymbol{V}_{22}^{\bar{z}}-U_{22} \boldsymbol{e}_{r^{\prime}} V_{22} \boldsymbol{e}_{\theta}+W_{22} \boldsymbol{e}_{z}, \quad \boldsymbol{V}_{20}-U_{20} \boldsymbol{e}_{i}+W_{20} \boldsymbol{e}_{\bar{z}}
$$

where

$\mathrm{i} U_{k l}, F_{k l}, \mathrm{i} W_{k l}, Q_{k l}, F_{k l}, Q_{02}, F_{02}$

and $Q_{00}$ are real for $(k, l)=(2,2),(2,0)$.

\section{APPENDIX C: A GALERKIN APPROXIMATION OF THE CASF EQUATIONS}

The solution of $(61)-(64)$ is projected into the vector space spanned by the following hydrodynamic cigenmodes, the first meridian one, the first azimuthal one and the first one exhibiting the azimuthal wave number $m=2$. Namely

$$
(\boldsymbol{u}, \hat{q})-Z_{1}\left(\boldsymbol{U}_{1}, \hat{Q}_{1}\right)+Z_{2}\left(\boldsymbol{U}_{2}, \hat{Q}_{2}\right)+\left[Z_{3}\left(\boldsymbol{U}_{3}, \hat{Q}_{3}\right)+\text { c.c. }\right] .
$$

where, for $j=1,2$, and $3,\left(\boldsymbol{U}_{j}, \hat{Q}_{j}\right)$ satisfies

$$
\begin{aligned}
& \boldsymbol{\nabla} \cdot \boldsymbol{U}_{j}-0, \quad \lambda_{j} U_{j}-\nabla \hat{Q}_{j}+R_{s}^{-1} \Delta \boldsymbol{U}_{j}-0 \text { in } \Sigma_{0}, \\
& \boldsymbol{U}_{j}-0 \quad \text { at } z-{ }^{\prime} \Lambda \\
& \boldsymbol{U}_{j} \cdot \boldsymbol{n}_{0}-\left[\left(\boldsymbol{\nabla} \boldsymbol{U}_{j}+\boldsymbol{\nabla} \boldsymbol{U}_{j}^{\top}\right) \cdot \boldsymbol{n}_{0}\right] \cdot\left(\boldsymbol{n}_{0} \times \boldsymbol{e}_{\theta}\right) \\
& \quad=\left[\left(\boldsymbol{\nabla} \boldsymbol{U}_{j} \mid \boldsymbol{\nabla} \boldsymbol{U}_{j}^{\top}\right) \cdot \boldsymbol{n}_{0}\right] \cdot \boldsymbol{e}_{\theta}=0 \quad \text { at } r=1,
\end{aligned}
$$

and the (real and negative) eigenvalues $\lambda_{1}, \lambda_{2}$, and $\lambda_{3}$ are the largest ones such that

$$
\begin{aligned}
& \boldsymbol{U}_{1}=U_{1} \boldsymbol{e}_{r} \mid W_{1} \boldsymbol{e}_{z}, \quad \boldsymbol{U}_{2}=V_{2} \boldsymbol{e}_{\theta} \\
& \text { and } \boldsymbol{U}_{3}-\mathrm{e}^{2 i \boldsymbol{\varphi}}\left(U_{3} \boldsymbol{e}_{i}+\mathrm{i} V_{3} \boldsymbol{e}_{\theta}+W_{3} \boldsymbol{e}_{z}\right),
\end{aligned}
$$

where the functions $U_{j}, V_{j}$, and $W_{j}$ are real and independent of $\theta$. In addition, the eigenmodes are taken such that

$$
\left\|U_{j}\right\|-1 \text { for } j-1,2,3,
$$

where $\|\cdot\|$ and $\langle\cdot, \cdot\rangle$ stand hereafter for the $L_{2}$-norm and $L_{2}$-inner product, respectively, i.e.,

$$
\|\boldsymbol{U}\|=\sqrt{\langle\boldsymbol{U}, \boldsymbol{U}\rangle}, \quad\langle\boldsymbol{U}, \boldsymbol{V}\rangle=\int_{\Sigma_{0}} \boldsymbol{U} \cdot \boldsymbol{V} d \boldsymbol{x}
$$

where the dot stands for the inner product (35). Note that (C5) can be imposed because the eigenvalues $\lambda_{1}, \ldots, \lambda_{3}$ are real and simple and the linear problem $(\mathrm{C} 2)-(\mathrm{C} 4)$ is equivariant under aximuthal reflections $\left(\theta \rightarrow-\theta, U_{j} \cdot e_{\theta} \rightarrow-U_{j} \cdot e_{\theta}\right)$ and under the symmetry $\boldsymbol{U}_{j} \rightarrow \overline{\boldsymbol{U}}_{j}, \hat{Q}_{j} \rightarrow \hat{Q}_{j}$. Also, because (C2)-(C4) is selfadjoint and the second mode is purcly arimuthal, the eigenfunctions satisfy

$$
\left\langle\boldsymbol{U}_{j}, \boldsymbol{U}_{k}\right\rangle-0 \text { if } j \neq k ; \quad \hat{Q}_{2}-0 .
$$

Note that since the original boundary conditions (63) and (64) are nonhomogeneous, and the eigenfunctions $U_{j}$ satisfy homogencous boundary conditions [sec (C3) and (C4)], convergence would be quite slow near the boundary if the whole series [expansion given by the nontruncated version of $(\mathrm{Cl})$ ] were considered. In that case, a change of variable should be made to take care of the nonhomogeneous boundary conditions before expanding the solution in terms of the hydrodynamic eigenfunctions. But this change of variable would yield much more involved equations than those obtained below, and the deficiency is not so important, at least in principle, in our rough truncation.

The evolution equations for the Galerkin coefficients $Z_{1}$, $Z_{2}$, and $Z_{3}$ are readily found [upon multiplication, with the inner product $(\mathrm{C} 7)$, of the second equation (62) by $\boldsymbol{U}_{j}$, integration by parts and substitution of (63) and (64), (C1)(C7)] to be

$$
\begin{aligned}
& Z_{1}^{\prime}-\lambda_{1} Z_{1}+\alpha_{122} Z_{2}^{2}+2 \alpha_{133}\left|Z_{3}\right|^{2}+\beta_{12}\left(|\beta|^{2}-|A|^{2}\right) Z_{2}, \\
& Z_{2}^{\prime}-\lambda_{2} Z_{2}+\alpha_{212} Z_{1} Z_{2}+\gamma\left(|B|^{2}-|A|^{2}\right), \\
& Z_{3}^{\prime}=\lambda_{3} Z_{3}\left|\alpha_{313} Z_{1} Z_{3}\right| \alpha_{323} Z_{2} Z_{3} \mid \beta_{33}\left(|B|^{2}\right.
\end{aligned}
$$

where

$$
\begin{aligned}
& \gamma-R_{s}^{-1} \int_{-\Lambda}^{\Lambda} e_{j} \cdot U_{2} \varphi_{S} d S, \quad \alpha_{j k l}-\mathcal{B}_{j}\left(\boldsymbol{U}_{k}, \boldsymbol{U}_{l}\right), \\
& \alpha_{j k l}=\mathcal{B}_{j}\left(\boldsymbol{U}_{k}, \overline{U_{l}}\right), \quad \beta_{j k}=\mathcal{B}_{j}\left(\boldsymbol{g}, \boldsymbol{U}_{k}\right) .
\end{aligned}
$$

Here, for $j=1, \ldots, 4$ and $m=1,2,3$, the bilinear functionals $\mathcal{B}_{j}$ and the (vector) function $\boldsymbol{\Phi}_{m}$ are defined as

$$
\mathcal{B}_{j}(\boldsymbol{U}, \boldsymbol{V})-\int_{\Sigma_{0}} \boldsymbol{U}_{j} \cdot(\boldsymbol{U} \times \boldsymbol{\nabla} \times \boldsymbol{V}) d \boldsymbol{x} .
$$

Note that, according to $(\mathrm{C} 5)$ and $(\mathrm{C} 12)-(\mathrm{C} 13)$, all coefficients in Eqs. (C9)-(C11) are real except $\alpha_{323}$ and $\beta_{33}$, which are purely imaginary, and

$$
\alpha_{122}+\alpha_{212}-0, \quad \alpha_{133}+\alpha_{313}-0 .
$$


Now we only need to use the variable $\hat{Z}_{3}$ $-Z_{3} \exp \left\{\mathrm{if} \int_{0}^{\tau}\left[\alpha_{32.3} Z_{2}(T)+\beta_{33}\left(|\beta(T)|^{2}-|A(T)|^{2}\right)\right] d T\right\} \quad 10$ rewrite Eqs. (C9) $-(\mathrm{C} 11)$ as

$$
\begin{aligned}
& Z_{1}^{\prime}=\lambda_{1} Z_{1}\left|\gamma_{2} Z_{2}^{2}\right| 2 \gamma_{3}\left|\hat{Z}_{3}\right|^{2} \mid \beta_{1}\left(|B|^{2} \quad|A|^{2}\right) Z_{2}, \\
& Z_{2}^{\prime}-\lambda_{2} Z_{2}-\gamma_{2} Z_{1} Z_{2}+\beta_{2}\left(|\beta|^{2}-|A|^{2}\right), \\
& Z_{3}^{\prime}-\lambda_{3} Z_{3}-\gamma_{3} Z_{1} Z_{3},
\end{aligned}
$$

where all coefficients $\gamma_{2}, \ldots, \gamma_{3}, \beta_{1}$, and $\beta_{2}$ are real.

Finally, a substitution of $(\mathrm{Cl})$ into the amplitude equalions (59) and (60) yiclds

$$
A^{\prime}(\tau)--(1+\mathrm{i} \widetilde{\delta}) A+\mathrm{i}\left(\alpha_{3}|A|^{2}+\alpha_{4}|B|^{2}\right) A-\mathrm{i} \gamma_{1} Z_{2} A+\mathrm{i} \widetilde{a},
$$

$$
B^{\prime}(\tau)--(1+\mathrm{i} \widetilde{\delta}) \beta+\mathrm{i}\left(\alpha_{3}|\beta|^{2}+\alpha_{4}|A|^{2}\right) B+\mathrm{i} \gamma_{1} Z_{2} \beta+\mathrm{i} \widetilde{a}
$$

where the real coefficient $\gamma_{1}$ is

$$
\gamma_{1}-\alpha_{5} \int_{\Sigma_{0}} g \cdot U_{2} d x
$$

${ }^{1}$ J. A. F. Plateau, "Sur les figures d'equilibre d'une masse liquide sans pesanteur," Mem. Acad. R. Belgique Nonv. Ser. 23, 5 (1849).

${ }^{2}$ J. A. F. Plateau, "Experimental and theoretical researches on the figures of equilibrium of a liquid mass withdrawn from the action of gravity," translation in Annu. Rep. Smithsonian Inst. 207 (1863).

${ }^{3}$ J. W. S. Lord Rayleigh, "On the instability of jets," Proc. London Math. Soe. 10, 4 (1879).

${ }^{4}$ J. W. S. I.ord Rayleigh, "On the stability of eylindrical fluid surfaces," Philos. Mag. 34, 145 (1892).

5X. Young, "Essay on the cohesion of fluids," Philos. Trans. R. Soc. London, Ser. A 306, 347 (1805).

${ }^{6}$ P. S. Laplace, Theory of Capillary Altractions, supplement of the tenth book on Celestial Mechanics (1832) (reprinted by Chelsea, New York; $1966)$.

${ }^{7} \mathrm{H}$. C. Kullmann, Thermocapillary Convection in Models of Crystal Growth (Springer-Verlag, Berlin, 1999).

${ }^{8} \mathrm{~J}$. Tsamopoulos, T. Y. Chen, and A. Borkar, "Viscous oscillations of capillary bridges," J. Fluid Mech. 235, 579 (1992).

${ }^{9}$ J. X. \%asadzinski, I. B. Sweency, H. T. Davis, and I. F.. Seriven, "Finite element calculation of fluid menisei and thin-films in model porous media," J. Colloid Interface Sci. 119, 108 (1987).

${ }^{10}$ T. Y. Chen, J. A. Tsamopoulos, and R. J. Good, "Capillary bridges between parallel and non-parallel surfaces and their stability," J. Colloid Interfice Sci. 151, 49 (1992).

${ }^{11} \mathrm{M}$. Higuera and .I. A. Nicolas, "I incar non-axisymmetric oscillations of ncarly-inviscid liquid bridges," Phys. Fluids 9, 276 (1997).

${ }^{12} F$. J. García and $A$. Castellanos, "One-dimensional models for slender ilxisymmetric viscous liquid bridges," Phys. Fluids 8, 2837 (1996).

${ }^{13} \mathrm{~T}$. Y. Chen and J. Tsamopoulos, "Vonlinear dynamies of capillary bridges: theory" J. Fluid Mech. 255, 373 (1993).

${ }^{14} \mathrm{~J}$. A. Vicolás and J. M. Vega, "Wcakly nonlincar oseillations of axisymmetric liquid bridges," J. Fluid Mech. 328, 95 (1996).

${ }^{15}$ F. J. Mancebo, J. $\Lambda$. Nicolas, and J. M. Vega, "Chrotic oscillations in a nearly-inviscid axisymmetric capillary bridge at $2: 1$ parametric resonance," Phys. Fluids 10, 1088 (1998)

${ }^{16} \mathrm{M}$. Jurish and $\mathrm{W}$. Löser, "Analysis of periodic non-rotational $W$ striations in $M_{0}$ single crystals due to non-steady thermocapillary convection," J. Cryst. Growth 102, 214 (1990).

${ }^{17}$ A. V. Anilkumar, R. N. Grugel, X. F. Shen, C. P. Lee, and T. G. Wang, "Control of thermocapillary conveetion in a liquid bridge by vibration;" J. Appl. Phys. 73. 4165 (1993).

${ }^{18} \mathrm{~J}$. A. Nicolís, D. Rivas, and J. M. Vega, "The interaction of thermocapillary convection and low-frequency vibration in nearly-inviscid liquid bridges," \%. Angew. Wath. Phys. 48, 389 (1997).

${ }^{19} \mathrm{~J}$. A. Nicolas, D. Rivas, and J. M. Vega, "On the steady streaming flow due to high frequency vibration in nearly-inviscid liquid bridges, " J. Fluid Mech. 354, 147 (1998).

${ }^{20}$ (i. K. Batchelor, An Iniroduction to fillid Dynamics (Cambridge I niversity Press, Cambridge, 1967).

${ }^{21}$ N. Riley, "Steady streaming," Annu. Rev. Fluid Mech. 33, 43 (2001).

${ }^{22}$ J. M. Voga, F. Knobloch, and C. Martel, "Ncarly inviscid Faraday waves in annular containers of moderately large aspect ratio," Pliysica D 154, $31.3(2001)$.

${ }^{2.3} \mathrm{C}$. Martel, J. A. Nicolís, and J. M. Vega, "Surface-wave damping in a brimful circular cylinder," J. Fluid Mech. 360, 213 (1998); see also, Corrigendum 373, 379 (1998).

${ }^{24} \mathrm{~J}$. Kevorkian and J. D. Cole, Perturbation Methods in Applied Mathematics (Springer-Verlag, New York, 1981).

${ }^{25}$ A. Sanz and J. L. Diez "Non-ixisymmetric oscillations of licpuid bridges," J. Fluid Mech. 205, 503 (1989)

${ }^{26}$ F. Lirsell, "Edge waves on al sloping beach," Proc. R. Soc. London, Ser. A 214, 79 (1952).

${ }^{27}$ M. Iliguerat, J. A. Nicolas, and J. M. Vega, "Linear oscillations of weakly dissipative axisymmetric liquid bridges," Plys. Fluids 6, 438 (1994).

${ }^{28} \mathrm{~J}$. A. Nicolas and J. W. Vega, "A note on the effeet of surface contamination in water wave damping," J. Fluid Mech. 410, 367 (2000).

${ }^{2 y}$ J. A. Vieolás and J. M. Vega, "I inear oseillations of axisymmetric viseous liquid bridges," Z. Angew. Math. Phys. 51, 701 (2000).

${ }^{31}$ J. Guckenheimer and P. Holmes, Nonlinear Oscillations Dynamical Systems and Bifincation of Vector Fields (Springer-Vorlag, Now York, 1983).

${ }^{31} \mathrm{~J}$. Miles and D. Henderson, "Parametrically forced surface waves," Annu. Rev. Fluid Mech. 22, 143 (1990).

${ }^{32}$ S. Fauve, "Parametric instabilities," in Dynamicis of Nonlinear and Disordered Systems, edited by G. Martinez Mekler and T. H. Seligman (World Scientific, Singapore, 1995), p. 67.

${ }^{33} \mathrm{E}$. Martin, C. Martel, and J. M. Vega, "Drift instability of standing Faraday waves in an annular container," J. Fluid Weeh (in press).

${ }^{34} Z$. C. Feng and P. R. Sethna, "Symmetry breaking bifurcations in resonant surface waves," J. Fluid Meeh. 199, 495 (1989).

${ }^{35} \mathrm{~F}$. Simonelli and J. P. Gollub, "Surface wave mode interactions: effects of symmetry and degeneracy," J. Fluid Mech. 199, 349 (1989). 